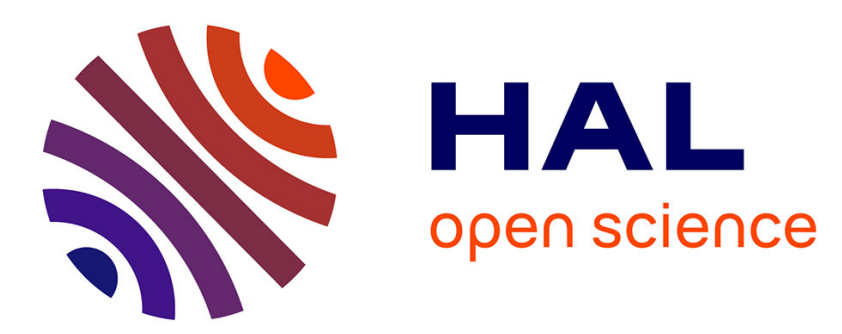

\title{
Coupled cluster singles and doubles polarization propagator accurate through the third order of Møller-Plesset theory
}

Tatiana Korona

\section{- To cite this version:}

Tatiana Korona. Coupled cluster singles and doubles polarization propagator accurate through the third order of Møller-Plesset theory. Molecular Physics, 2010, 108 (03-04), pp.343-357. 10.1080/00268970903476654 . hal-00580672

\section{HAL Id: hal-00580672 \\ https://hal.science/hal-00580672}

Submitted on 29 Mar 2011

HAL is a multi-disciplinary open access archive for the deposit and dissemination of scientific research documents, whether they are published or not. The documents may come from teaching and research institutions in France or abroad, or from public or private research centers.
L'archive ouverte pluridisciplinaire HAL, est destinée au dépôt et à la diffusion de documents scientifiques de niveau recherche, publiés ou non, émanant des établissements d'enseignement et de recherche français ou étrangers, des laboratoires publics ou privés. 


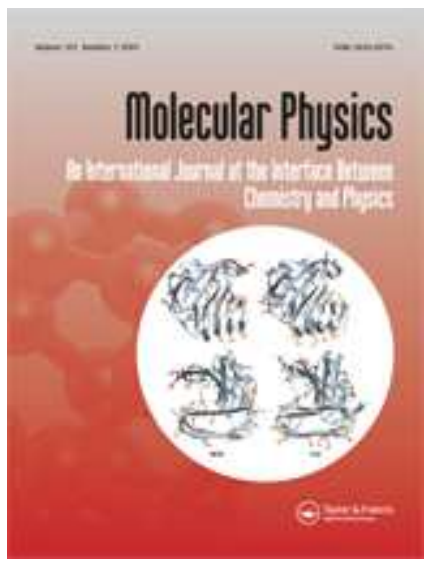

\section{Coupled cluster singles and doubles polarization propagator accurate through the third order of Møller-Plesset theory}

\begin{tabular}{|c|c|}
\hline Journal: & Molecular Physics \\
\hline Manuscript ID: & TMPH-2009-0264.R1 \\
\hline Manuscript Type: & Special Issue Paper - In honour of Prof Werner 60th birthday \\
\hline $\begin{array}{r}\text { Date Submitted by the } \\
\text { Author: }\end{array}$ & 07-Nov-2009 \\
\hline Complete List of Authors: & Korona, Tatiana; University of Warsaw, Faculty of Chemistry \\
\hline Keywords: & $\begin{array}{l}\text { coupled cluster theory, molecular properties, polarization } \\
\text { propagator, linear response function }\end{array}$ \\
\hline \multicolumn{2}{|c|}{$\begin{array}{l}\text { Note: The following files were submitted by the author for peer review, but cannot be converted } \\
\text { to PDF. You must view these files (e.g. movies) online. }\end{array}$} \\
\hline TMPH-2009-0264.R1.zip & \\
\hline
\end{tabular}

\section{scholarONE" \\ Manuscript Central}


Molecular Physics

Vol. 00, No. 00, Month 200x, 1-21

\title{
RESEARCH ARTICLE
}

\section{Coupled cluster singles and doubles polarization propagator accurate through the third order of Møller-Plesset theory}

\author{
Tatiana Korona \\ Faculty of Chemistry, University of Warsaw, ul. Pasteura 1, 02-093 Warsaw, Poland
}

(Received 00 Month 200x; final version received 00 Month 200x)

\begin{abstract}
A noniterative low-cost correction for the polarization propagator of coupled cluster theory limited to single and double excitations (CCSD) has been developed. The proposed expression accounts for the most important terms resulting from triple excitation operators, which are missing in the CCSD formula. These contributions have been identified and analysed for both existing theories of coupled cluster response functions, i.e. for the time-dependent and expectation-value approaches. It has been demonstrated on the operator level that - by an appropriate rearrangement of these terms and an elimination of long energy denominators the total third-order contribution can be recast into the form, which does not contain triple excitation operators, but is still correct through the third order of Møller-Plesset theory. The computational cost of the proposed correction scales with the sixth power of the molecular size, and is therefore at least one order of magnitude smaller than in the case of an explicit inclusion of triple excitation operators into the propagator formula.
\end{abstract}

Keywords: Coupled cluster theory, molecular properties, polarization propagator, linear response function

\section{Introduction}

Coupled cluster (CC) theory [1] has become one of the most popular tools for a description of dynamic electron correlation in molecules. For instance, the CCSD $(\mathrm{T})$ method (CC theory limited to single and double excitations with a noniterative inclusion of triple excitations) [2] has been denoted as a 'golden standard' of quantum chemistry for small-size systems. A notable feature of CC theory is its sizeextensivity $[3,4]$, which is also preserved in truncated CC models. A success of $\mathrm{CC}$ theory in describing dynamic electron correlation for the energy creates expectations for a similarly good description of molecular properties. Contrary to the correlation energy, some of these properties, like dipole moments and polarizabilities, can be directly compared to experimental values. It is well known that the development of $\mathrm{CC}$ methods for describing the molecular properties requires a lot of additional effort in comparison to the calculation of the CC correlation energy. The main difficulty arises from the nonvariational and projective character of the CC equations [5] (for instance, the CC energy is not manifestly real, if a truncated cluster operator $T$ is used). One can deal with the signalized problems in two inequivalent ways, which can be in general denoted as derivative and expectationvalue methods, respectively. It is noteworthy that both approaches give the same results in the limit of full CC theory.

Email:tatiana.korona@chem.uw.edu.pl, phone: +48-228220211 ext. 207, fax: +48-228222309

Dedicated to Professor Hans-Joachim Werner on the occasion of his 60th birthday

ISSN: 0040-5167 print/ISSN 1754-2278 online

(C) 200x Taylor \& Francis

DOI: $10.1080 / 0040516 Y Y x x x x x x x x$

http://www.informaworld.com 
The first method, which has become a standard nowadays, has been pioneered by Monkhorst [6] and Dalgaard and Monkhorst [7] and is based on an analytic differentiation of the CC energy followed by an elimination of the derivative amplitudes [8-11] or alternatively on the use of the CC energy Lagrangian $[12,13]$ [giving the approach known as gradient or linear-response (LR) CC theory]. This method has been extended to dynamic properties by the use of a time-dependent pseudoenergy [6, 7], and is therefore denoted frequently as time-dependent (TD) CC theory of molecular properties. The development of the TD-CC approach has been greatly facilitated by an introduction of the time-dependent CC pseudoenergy Lagrangian [14-16]. In the last 15 years the implementations of TD-CC2 [17], TD-CCSD [16], orbital-relaxed TD-CCSD [18], TD-CC3 [19], and even TD-CCSDT [20] and arbitrary order [21] frequency-dependent linear-response properties (also called polarization propagators [22]) and their numerous applications (see e.g. Refs. [23-30]) have been reported in the literature. It should be noted that although the orbital-relaxed TD-CCSD approach has been recently developed [18], the standard nonrelaxed version of TD-CC theory should be rather used, since the relaxation of orbitals results in an additional set of poles (generated by the coupled Hartree-Fock equations) for real frequencies [16].

An approach alternative to TD-CC makes a direct use of the basic quantum mechanics expression for the average value of some operator (or its generalizations for higher-order properties) with the exact wave function replaced by its normalized CC counterpart, and will be called shortly XCC theory in this paper. The development of this method has been hindered for a long time by complications stemming from the presence of the deexcitation operator $T^{\dagger}$ (an adjoint of the usual cluster operator $T$ of CC theory), resulting in an infinite series of products of $T^{\dagger}$ and $T$ (see e.g. Ref. [31]). The solution to this problem — proposed some time ago by Jeziorski and Moszynski [32] — relies on the use of a new excitation operator $S$ (vide infra). In the same publication general formulae for the first-order expectation-value CC properties have been proposed. Recently, this approach has been extended to dynamic second-order properties [33]. The corresponding polarization propagator for CC theory limited to single and double excitations (CCSD) has been derived and implemented in Ref. [34]. It should be emphasized that the $\mathrm{XCC}$ approach does not require a costly calculation of the left-hand solution of CC equations (the so-called $\Lambda$ vector [8]), so the XCC first-order properties can be calculated at virtually no cost, once the $T$ operator is available [35]. In addition, $\mathrm{XCC}$ theory makes it possible to introduce cumulants of the reduced two-electron density matrices [36] and it should be used in all cases where properly defined CC cumulants are required, like in the expressions for the exchange energy contributions [37-39] of symmetry-adapted perturbation theory (SAPT) of intermolecular interactions [40].

The exact polarization propagator exhibits various symmetry properties, like Hermitian (or time-reversal for purely real or purely imaginary perturbations) and operator exchange symmetries. The time-reversal symmetry is not fulfilled by the TD-CC polarization propagator in its original formulation [14], while the XCC propagator satisfies by definition the Hermitian and time-reversal symmetries, but the truncated forms of this propagator show small (high-order) violations of the operator exchange symmetry [33].

Both TD-CC and XCC formulae for polarization propagators include some terms containing connected triple excitations, which contribute already in the third order of Møller-Plesset (MP) theory. As a result, the TD-CCSD and XCCSD methods for the second-order properties are rigorously correct through the second MP order only $[16,33,41]$. The aim of this paper is to derive a low-cost formula accounting for 
the missing MP3 contributions by employing the operator techniques, which enable the elimination of long triple energy denominators, and to propose an extension of this formula for the nonperturbative case.

\section{TD-CC and XCC propagators}

General expressions for TD-CC and XCC propagators have been developed elsewhere $[14,33]$. In this section only the final formulae in a form suitable for the further analysis will be reviewed. For a convenience a scalar product notation: $\langle X\rangle=\langle\Phi \mid X \Phi\rangle$ and $\langle X \mid Y\rangle=\langle X \Phi \mid Y \Phi\rangle$ (where $\Phi$ is the reference determinant) will be used. Operators $X$ and $Y$ acting as perturbing operators for second-order properties are assumed to be Hermitian one-electron operators throughout this paper.

\subsection{TD-CC polarization propagator}

A general formula for the TD-CC propagator for the Hamiltonian $H$, perturbing operators $X, Y$, and for $\omega$ being a frequency of the perturbing field, is given by the following equation [14]

$$
\langle\langle X ; Y\rangle\rangle_{\omega}=\langle 1+\Lambda \mid[\bar{Y}, \Omega(\omega)]\rangle+\langle 1+\Lambda \mid[\bar{X}, \Xi(-\omega)]\rangle+\langle 1+\Lambda \mid[[\bar{H}, \Omega(\omega)], \Xi(-\omega)]\rangle,
$$

where $\bar{Z}$ denotes a similarity-transformed operator

$$
\bar{Z}=e^{-T} Z e^{T} .
$$

An excitation operator $\Lambda$, which appears already in the formulation of the firstorder LR-CC properties $[8,9,12,13]$, is defined as a solution of the following equation

$$
\langle 1+\Lambda \mid[\bar{H}, \tau]\rangle=0
$$

where $\tau$ can be any excitation operator within a considered excitation range [e.g. in the present study excitations are limited to singles, doubles and triples (CCSDT)]. A first-order operator $\Omega(\omega)$ describes the response of a molecule to the perturbing field $X$ oscillating with a frequency $\omega$ (note that $\Omega(\omega)$ corresponds to $t^{X}(-\omega)$ in Ref. [16]). The operator $\Omega(\omega)$ can be obtained by solving the following linear equation

$$
\langle\tau \mid \bar{X}\rangle+\langle\tau \mid[\bar{H}, \Omega(\omega)]\rangle+\omega\langle\tau \mid \Omega(\omega)\rangle=0
$$

An analogous equation for $\Xi(-\omega)$ can be retrieved from Eq. (4) by replacing $X$ by $Y$ and $\omega$ by $-\omega$.

The fact that Eq. (1) does not fulfill the time-reversal symmetry has been realizednoticed already in Ref. [14]. In order to force the correct symmetry usually a symmetrized form of Eq. (1) is usually utilized, as proposed in Ref. [15]. This solution has a side-effect that some high-order contributions (in terms of the MP fluctuation operator $W=H-F$, where $F$ is the Fock operator) appear with a wrong coefficient of $\frac{1}{2}$ [33], what however does not lead to any known practical problems. 
If the excitations are restricted to singles, doubles and triples and the nested commutator expansion

$$
e^{-T} Z e^{T}=Z+[Z, T]+\frac{1}{2 !}[[Z, T], T]+\ldots,
$$

is utilized, then a rather lengthy formula for the TD-CCSDT propagator results from Eq. (1). Since the present study is focused on the analysis of the low-order parts of the TD-CCSDT propagator, only the contributions relevant to the further derivations will be considered. These contributions are listed explicitly in Table 1 . The same table also reports the lowest order of MP theory, to which a given term contributes in the propagator expression. Finally, the table shows whether listed terms are included already on the TD-CCSD level. It follows from an examination of Table 1 that there is one contribution which is entirely missing, while two more terms are included only partially if triple excitations are neglected. The latter terms miss the triples contributions, which enter indirectly through the single and double excitation operators. Therefore, the TD-CCSD polarization propagator is rigorously correct only through the second order of MP theory [16].

\subsection{XCC polarization propagator}

An alternative approach for the coupled cluster second-order properties has been presented recently by Moszynski et al. [33]. This method is based on a general definition of the polarization propagator [22],

$$
\langle\langle X ; Y\rangle\rangle_{\omega}=-\sum_{n=1}^{\infty} \frac{\left\langle\Psi_{0} \mid Y \Psi_{n}\right\rangle\left\langle\Psi_{n} \mid X \Psi_{0}\right\rangle}{E_{n}-E_{0}+\omega}-\sum_{n=1}^{\infty} \frac{\left\langle\Psi_{0} \mid X \Psi_{n}\right\rangle\left\langle\Psi_{n} \mid Y \Psi_{0}\right\rangle}{E_{n}-E_{0}-\omega}
$$

where $E_{k}$ and $\Psi_{k}$ are eigenvalues and eigenfunctions for the $k$ th electronic state, respectively. It is noteworthy that the second term of the r.h.s. of Eq. (6) can be obtained from the first one either by taking its complex conjugate followed by replacing of $\omega$ by $-\omega^{*}$ (the operation called generalized complex conjugate - g.c.c. in Ref. [33], which is related to the time-reversal symmetry), or by exchanging $X$ and $Y$ operators and replacing of $\omega$ by $-\omega$ (the operation called operator exchange - op.ex.). In the approach of Moszynski et al. [33] one utilizes the normalized CC wave function as $\Psi_{0}$

$$
\Psi_{0}=\frac{e^{T} \Phi}{\left\langle e^{T} \mid e^{T}\right\rangle^{1 / 2}},
$$

and the following Ansatz for the first-order wave function $\Psi^{(1)}$, being a linear response to the perturbation $X$ oscillating with a frequency $\omega$

$$
\Psi^{(1)}=\frac{\left(\Omega_{0}(\omega)+\Omega(\omega)\right) e^{T} \Phi}{\left\langle e^{T} \mid e^{T}\right\rangle^{1 / 2}} .
$$

In Eq. (8) $\Omega_{0}(\omega)$ is a number (which does not enter the final equations and does not need to be considered any further) and $\Omega(\omega)$ is an excitation operator defined by Eq. (4). Additionally, an excitation operator $S$, appearing for the first time in expectation-value CC theory for the first-order properties [32], will beis used in the XCC polarization propagator formula. This operator is uniquely defined by 
the equation

$$
e^{S} \Phi=\frac{e^{T^{\dagger}} e^{T} \Phi}{\left\langle e^{T} \mid e^{T}\right\rangle}
$$

It has been proven $[32,42]$ that the $S$ operator is connected (it can be constructed from a finite number of multiple commutators containing $T$ and $T^{\dagger}$ ), what makes it a useful quantity for constructing of size-extensive models. The $S$ operator correct through the third $W$ order is given by the equations [32]

$$
\begin{aligned}
S_{1} & =T_{1}+\widehat{\mathcal{P}}_{1}\left(\left[T_{1}^{\dagger}, T_{2}\right]+\left[T_{2}^{\dagger}, T_{3}\right]\right), \\
S_{2} & =T_{2}+\frac{1}{2} \widehat{\mathcal{P}}_{2}\left(\left[\left[T_{2}^{\dagger}, T_{2}\right], T_{2}\right]\right), \\
S_{3} & =T_{3} .
\end{aligned}
$$

Starting from Eqs. (6)-(9) the following formula has been derived in Ref. [33] for the $\mathrm{CC}$ polarization propagator

$$
\langle\langle X ; Y\rangle\rangle_{\omega}=\left\langle e^{-S} e^{T^{\dagger}} Y e^{-T^{\dagger}} e^{S} \mid \widehat{\mathcal{P}}\left(e^{S^{\dagger}} \Omega(\omega) e^{-S^{\dagger}}\right)\right\rangle+\text { g.c.c. }
$$

In Eq. (11) $\widehat{\mathcal{P}}$ stands for the superoperator $\widehat{\mathcal{P}}_{1}+\widehat{\mathcal{P}}_{2}+\widehat{\mathcal{P}}_{3}+\ldots$, defined such that $\widehat{\mathcal{P}}_{n}$ "projects" the $n$-tuple excitation part of an operator $Z$, i.e.,

$$
\widehat{\mathcal{P}}_{n}(Z)=(n !)^{-2} \sum_{i_{1} \ldots i_{n}} \sum_{a_{1} \ldots a_{n}}\left\langle e_{a_{n} \ldots a_{1}}^{i_{1} \ldots i_{n}} Z\right\rangle e_{i_{1} \ldots i_{n}}^{a_{n} \ldots a_{1}},
$$

and $e_{i_{1} \ldots i_{n}}^{a_{n} \ldots a_{1}}=a^{a_{1}} \cdots a^{a_{n}} a_{i_{n}} \cdots a_{i_{1}}$ is the $n$-body spinorbital excitation operator $\left(a^{a_{k}}\right.$ and $a_{i_{k}}$ are a virtual spinorbital creation operator and an occupied spinorbital annihilation operator, respectively). The form of Eq. (11) makes it straightforward to apply the expansion from Eq. (5). The resulting formula contains a finite, although extremely long series of multiple commutators of the $S^{\dagger}$ and $T$ operators. Most of these terms give a very small contribution to the total value of the polarization propagator and can be safely neglected. The resulting truncated models are also size-extensive since they are constructed as the sums of multiple commutators of connected quantities. The usual criteria for the importance of such terms are based on the analysis of the orders of $T$ 's and $W$ 's [33]. These criteria have been used to develop several truncation schemes for the CCSD case [34], from which the $\operatorname{CCSD}(3)$ model has been selected as the optimal one for many-electron cases. The CCSD(3) model includes a minimum number of terms, which are necessary to account for all contributions up to the $\mathcal{O}\left(W^{3}\right)$ order arising from Eq. (11), if $T, \Omega$, and $\Xi$ are truncated after single and double excitations. It is important to emphasize that this model does not include all contributions of the $\mathcal{O}\left(W^{3}\right)$ order entering the exact XCC polarization propagator. Similarly as for the TD-CCSD case, missing terms arise from triple excitation operators $T_{3}, \Omega_{3}(\omega)$, and $\Xi_{3}(-\omega)$.

All components of the XCCSDT polarization propagators giving a contribution of the $\mathcal{O}\left(W^{3}\right)$ or lower order are shown in Table 2. As in Table 1, the leading MP order and the presence of a given term in the XCCSD (equivalent to the $\operatorname{CCSD}(3)$ model from Ref. [34]) formula are also indicated in this table.

Some contributions presented in Tables 1 and 2 look quite different. However, it can be shown by an order-by-order MP analysis that they contain the same or very similar contributions through the third MP order. 


\section{CC polarization propagator correct through the third MP order}

It is clear from Tables 1 and 2 that triple excitation operators are necessary, if a propagator correct through the third MP order is required. Obviously, the presence of triple excitations increases the computational cost of the $\mathrm{CC}$ propagator from the sixth to at least the seventh power of the molecular size $\mathcal{N}$, what in turn reduces substantially sizes of molecules for which such calculations can be performed. In this section it will be shown how to introduce the corrections to XCCSD and TD-CCSD polarization propagators, which account for missing $\mathcal{O}\left(W^{3}\right)$ contributions, but do not contain the $T_{3}, \Omega_{3}(\omega)$, or $\Xi_{3}(-\omega)$ excitation operators.

\subsection{Møller-Plesset expansion of excitation operators}

An analysis of the contributions from Tables 1 and 2 is based on the Møller-Plesset expansion of the excitation operators. Such expansions can be most easily obtained from the following iterative formulae [32, 33, 43]

$$
\begin{aligned}
T_{n} & =\widehat{\mathcal{R}}_{n, 0}\left(e^{-T} W e^{T}\right), \\
\Omega_{n}(\omega) & =\widehat{\mathcal{R}}_{n, \omega}\left(e^{-T} X e^{T}+\left[e^{-T} W e^{T}, \Omega(\omega)\right]\right) .
\end{aligned}
$$

The action of the resolvent superoperator $\widehat{\mathcal{R}}_{n, \omega}$ on znsome operator $Z A$ is given by the equation

$\widehat{\mathcal{R}}_{n, \omega}(\mathcal{Z} A)=(n !)^{-2} \sum_{i_{1} \ldots i_{n}} \sum_{a_{1} \ldots a_{n}}\left(\epsilon_{i_{1}}+\ldots+\epsilon_{i_{n}}-\epsilon_{a_{1}}-\ldots-\epsilon_{a_{n}}-\omega\right)^{-1}\left\langle e_{a_{n} \ldots a_{1}}^{i_{1} \ldots i_{n}} \bigotimes\langle\lambda\rangle e_{i_{1} \ldots i_{n}}^{a_{n} \ldots a_{1}}\right.$,

where $\epsilon_{p}$ is a spinorbitalan energy forof a spinorbital $p$. As shown in Ref. [33], the $\widehat{\mathcal{R}}_{n, \omega}$ superoperator fulfills the identity (for any operator $A$ )

$$
\left[F, \widehat{\mathcal{R}}_{n, \omega}(X A)\right]+\omega \widehat{\mathcal{R}}_{n, \omega}(X A)+\widehat{\mathcal{P}}_{n}(X A)=0
$$

By iterating Eq. (13) the following expressions can be obtained for consecutive orders of $T^{(k)}$ and $\Omega^{(k)}(\omega)$, where $k$ denotes the power of the fluctuation operator $W$

$$
\begin{aligned}
T_{2}^{(1)} & =\widehat{\mathcal{R}}_{2,0}(W), \\
T_{n}^{(2)} & =\widehat{\mathcal{R}}_{n, 0}\left(\left[W, T_{2}^{(1)}\right]\right), \quad n=1,2,3, \\
T_{n}^{(3)} & =\widehat{\mathcal{R}}_{n, 0}\left(\left[W, T_{1}^{(2)}+T_{2}^{(2)}+T_{3}^{(2)}\right]+\left(1-\delta_{1 n}\right) \frac{1}{2}\left[\left[W, T_{2}^{(1)}\right], T_{2}^{(1)}\right]\right), \quad n=1,2,3, \\
\Omega_{1}^{(0)}(\omega) & =\widehat{\mathcal{R}}_{1, \omega}(X), \\
\Omega_{n}^{(1)}(\omega) & =\widehat{\mathcal{R}}_{n, \omega}\left(\left[X, T_{2}^{(1)}\right]+\left[W, \Omega_{1}^{(0)}(\omega)\right]\right), \quad n=1,2, \\
\Omega_{n}^{(2)}(\omega) & =\widehat{\mathcal{R}}_{n, \omega}\left(\left[X, T_{n}^{(2)}+T_{n+1}^{(2)}\right]+\left[W, \Omega_{1}^{(1)}(\omega)+\Omega_{2}^{(1)}(\omega)\right]+\left[\left[W, T_{2}^{(1)}\right], \Omega_{1}^{(0)}(\omega)\right]\right), \quad n=1,2, \\
\Omega_{3}^{(2)}(\omega) & =\widehat{\mathcal{R}}_{3, \omega}\left(\left[X, T_{3}^{(2)}\right]+\left[W, \Omega_{2}^{(1)}(\omega)\right]+\left[\left[W, T_{2}^{(1)}\right], \Omega_{1}^{(0)}(\omega)\right]+\frac{1}{2}\left[\left[X, T_{2}^{(1)}\right], T_{2}^{(1)}\right]\right), \\
\Omega_{1}^{(3)}(\omega) & =\widehat{\mathcal{R}}_{1, \omega}\left(\left[X, T_{1}^{(3)}+T_{2}^{(3)}\right]+\left[W, \Omega_{1}^{(2)}(\omega)+\Omega_{2}^{(2)}(\omega)+\Omega_{3}^{(2)}(\omega)\right]+\right. \\
& \left.+\left[\left[W, T_{2}^{(2)}\right], \Omega_{1}^{(0)}(\omega)\right]+\left[\left[W, T_{1}^{(2)}\right], \Omega_{1}^{(0)}(\omega)\right]+\left[\left[W, T_{2}^{(1)}\right], \Omega_{1}^{(1)}(\omega)\right]\right) .
\end{aligned}
$$


The formulae for $\Omega^{(k)}(\omega)(k \leq 2)$ and for $T^{(k)}$ have been already presented elsewhere and are only repeated for the sake of completeness. Note that in Eq. (41) of Ref. [33] the last term is missing in the definition of $\Omega_{3}^{(2)}(\omega)$. The expressions for $\Xi_{n}^{(k)}(-\omega)$ are analogous to $\Omega_{n}^{(k)}(\omega)$ and will be not listed here.

The $\Lambda_{n}$ operator can be expanded in terms of the $n$-body excitation operators $e_{i_{1} \ldots i_{n}}^{a_{1} \ldots a_{n}}$, denoted here shortly as $\tau_{K(n)}$,

$$
\Lambda_{n}=\sum_{K(n)} \Lambda_{K(n)} \tau_{K(n)}
$$

The iterative formula for the expansion coefficient $\Lambda_{K(n)}$ has the following form

$$
\Lambda_{K(n)}=\left(\Delta \epsilon_{K(n)}\right)^{-1}\left\langle 1+\Lambda \mid\left[\bar{W}, \tau_{K(n)}\right]\right\rangle
$$

where $\Delta \epsilon_{K(n)}$ is the energy denominator from Eq. (14) for $\omega=0$. By iterating Eq. (18) one can easily verify that the $\Lambda$ and $T$ operators have the same MP expansion up to the second MP order and that differences between them appear first on the MP3 level

$$
\begin{aligned}
\Lambda_{2}^{(1)} & =T_{2}^{(1)} \\
\Lambda_{n}^{(2)} & =T_{n}^{(2)}, \quad n=1,2,3 \\
\Lambda_{K(1)}^{(3)} & =\left(\Delta \epsilon_{K(1)}\right)^{-1}\left\{\left\langle\left[\left[W, T_{1}^{(2)}\right], \tau_{K(1)}\right]\right\rangle+\left\langle T_{2}^{(1)} \mid\left[\left[W, T_{2}^{(1)}\right], \tau_{K(1)}\right]\right\rangle+\right. \\
& \left.+\left\langle T_{1}^{(2)} \mid\left[W, \tau_{K(1)}\right]\right\rangle+\left\langle T_{2}^{(2)} \mid\left[W, \tau_{K(1)}\right]\right\rangle\right\} .
\end{aligned}
$$

It is important to observe that no contribution from the triple excitation operator $T_{3}^{(2)}$ enters the expression for $\Lambda_{1}^{(3)}$.

Since for the XCC propagator also the $S$ operator is needed, its MP expansion is also of interest here. It can be deduced from Eq. (10) that the $S_{n}$ and $T_{n}$ operators are identical through the $\mathcal{O}\left(W^{2}\right)$ order. FThe first differences between $S$ and $T$ occur in the third MP order, when terms resulting from the commutator expansion containing the $T^{\dagger}$ deexcitation operator start to contribute to $S$,

$$
\begin{aligned}
S_{1}^{(3)} & =T_{1}^{(3)}+\widehat{\mathcal{P}}_{1}\left(\left[\left(T_{1}^{(2)}\right)^{\dagger}, T_{2}^{(1)}\right]+\left[\left(T_{2}^{(1)}\right)^{\dagger}, T_{3}^{(2)}\right]\right), \\
S_{2}^{(3)} & =T_{2}^{(3)}+\frac{1}{2} \widehat{\mathcal{P}}_{2}\left(\left[\left[\left(T_{2}^{(1)}\right)^{\dagger}, T_{2}^{(1)}\right], T_{2}^{(1)}\right]\right) .
\end{aligned}
$$

From an analysis of Eqs. (16)-(20) one can identify fragments of single and double excitation operators dependent on the triple excitation operators $T_{3}^{(2)}$ and $\Omega_{3}^{(2)}(\omega)$. The explicit expressions for these operator fragments are listed below (the small letter $t$ in superscript denotes that only triple-related parts of the operator are taken into account)

$$
\begin{aligned}
T_{n}^{(3 t)} & =\widehat{\mathcal{R}}_{n, 0}\left(\left[W, T_{3}^{(2)}\right]\right), \quad n=1,2, \\
\Omega_{2}^{(2 t)}(\omega) & =\widehat{\mathcal{R}}_{2, \omega}\left(\left[X, T_{3}^{(2)}\right]\right), \\
\Omega_{1}^{(3 t)}(\omega) & =\widehat{\mathcal{R}}_{1, \omega}\left(\left[X, T_{1}^{(3 t)}+T_{2}^{(3 t)}\right]+\left[W, \Omega_{2}^{(2 t)}(\omega)+\Omega_{3}^{(2)}(\omega)\right]\right), \\
S_{1}^{(3 t)} & =T_{1}^{(3 t)}+\widehat{\mathcal{P}}_{1}\left(\left[\left(T_{2}^{(1)}\right)^{\dagger}, T_{3}^{(2)}\right]\right) .
\end{aligned}
$$


As already mentioned, $\Lambda_{1}^{(3 t)}=0$. By substituting operators from Eq. (21) into the terms from Tables 1 and 2 it is now easy to select the contributions which depend on triple excitation operators and are therefore not included into the XCCSD and TD-CCSD propagators.

\section{2. $\quad$ The elimination of triple excitation operators from the MP3 contributions of the XCC polarization propagator}

The MP3 contributions of the XCC polarization propagator, missing within the XCCSD treatment are collected in Table 3. In Ref. [32] it has been demonstrated that long triple denominators from the MP-expanded expression for the first-order CC properties orders can be eliminated on the operator level. Similar manipulations with double excitation operators have been utilized for the XCC propagator in order to separate true and apparent correlation contributions up to the second MP order [33]. The elimination of long energy denominators can be most easily explained on the algebraic level (see e.g. Ref. [44]). If a sum of two similar expressions is computed, which differ only by various placements of the energy denominators, and if the denominators for the first and second contribution have the form $a^{-1}(a+b)^{-1}$ and $b^{-1}(a+b)^{-1}$, respectively ( $a$ and $b$ denote sums of orbital energies), then the sum of these two denominators equals simply to $a^{-1} b^{-1}$, i.e. the long $(a+b)^{-1}$ denominator dissapears. Such techniques have been used to derive various orbital expressions, like polarizabilities for the second order of MP theory [45]. An important advantage of the approach presented in Refs. [32, 33] consists in performing such eliminations on the operator level, i.e. without the necessity of keeping track of a multitude of algebraic expressions, which would appear if the Wick theorem or the contraction theorem is applied to the contributions collected in Table 3. In the remainder of this section this approach will be extended and applied to eliminate the $T_{3}^{(2)}$ and $\Omega_{3}^{(2)}(\omega)$ operators from the MP3 contributions to the XCCSDT and TD-CCSDT propagators. In order to facilitate the search for appropriate pairs of terms from Table 3 a diagrammatic analysis can be utilized. A short description of this technique, as well as diagrammatic representations of most important derivation steps from the next Section, are presented in the Appendix.

\subsubsection{Terms Ia, Id, IVa, VII}

Let us start from two terms containing the perturbed triple excitation operator $\Omega_{3}^{(2)}(\omega)$. The $\Omega_{3}^{(2)}(\omega)$ operator can be rewritten in a short form as

$$
\Omega_{3}^{(2)}(\omega)=\widehat{\mathcal{R}}_{3, \omega}(Z)
$$

where the operator $Z$ is given by the equation

$$
Z=\left[X, T_{3}^{(2)}\right]+\left[W, \Omega_{2}^{(1)}(\omega)\right]+\left[\left[W, T_{2}^{(1)}\right], \Omega_{1}^{(0)}(\omega)\right]+\frac{1}{2}\left[\left[X, T_{2}^{(1)}\right], T_{2}^{(1)}\right]
$$

The operator $\Omega_{3}^{(2)}(\omega)$ appears in the terms Id and VII. The term Id can be rewritten as follows

$$
\begin{array}{r}
\operatorname{Id}=\left\langle Y \mid \widehat{\mathcal{R}}_{1, \omega}\left(\left[W, \Omega_{3}^{(2)}(\omega)\right]\right)\right\rangle=\left\langle\widehat{\mathcal{R}}_{1, \omega^{*}}(Y) \mid\left[W, \Omega_{3}^{(2)}(\omega)\right]\right\rangle= \\
=\left\langle\widehat{\mathcal{P}}_{2}(W) \widehat{\mathcal{R}}_{1, \omega^{*}}(Y) \mid \Omega_{3}^{(2)}(\omega)\right\rangle=\left\langle\widehat{\mathcal{P}}_{2}(W) \widehat{\mathcal{R}}_{1, \omega^{*}}(Y) \mid \widehat{\mathcal{R}}_{3, \omega}(Z)\right\rangle .
\end{array}
$$


Here the "Hermicity" of the superresolvent operator has been used ( $A$ and $B$ denote some general operators)

$$
\left\langle\mathrm{X} A \mid \widehat{\mathcal{R}}_{n, \omega}(\mathrm{K} B)\right\rangle=\left\langle\widehat{\mathcal{R}}_{n, \omega^{*}}(\mathrm{X} A) \mid \mathrm{K} B\right\rangle,
$$

as well as the facts that (i) the commutator $\left[W, \Omega_{3}^{(2)}(\omega)\right]$ can be replaced by the product $W \Omega_{3}^{(2)}(\omega)$ (note that $-\left|\Omega_{3}^{(2)}(\omega) W\right\rangle$ generates a pentuple excitation, which obviously cannot be deexcited by a single $\widehat{\mathcal{R}}_{1, \omega^{*}}(Y)$ operator), and (ii) the $W$ operator should deexcite two out of three excitations produced by the $\Omega_{3}^{(2)}(\omega)$ operator.

The second term containing the $\Omega_{3}^{(2)}(\omega)$ operator can be rewritten as

$$
\mathrm{VII}=\left\langle T_{2}^{(1)} \mid\left[Y, \Omega_{3}^{(2)}(\omega)\right]\right\rangle=\left\langle T_{2}^{(1)} \widehat{\mathcal{P}}_{1}(Y) \mid \Omega_{3}^{(2)}(\omega)\right\rangle=\left\langle\widehat{\mathcal{R}}_{2,0}(W) \widehat{\mathcal{P}}_{1}(Y) \mid \widehat{\mathcal{R}}_{3, \omega}(Z)\right\rangle,
$$

if one makes use of the Hermicity of the $Y$ operator and notices that in this case $Y$ plays a role of a single deexcitation operator. In the last step the definitions of the $T_{2}^{(1)}$ and $\Omega_{3}^{(2)}(\omega)$ operators (Eqs. (16) and (22), respectively) have been used. Note that the operators on the bra side of Eq. (26) commute, since they are both the excitation operators.

In order to continue the following identity should be employed

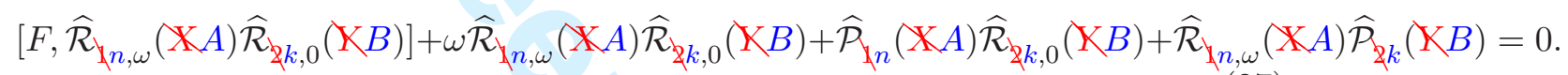

Eq. (27) has been derived by performing an addition of two equations: the first one obtained by the multiplication of Eq. (15) by $\widehat{\mathcal{R}}_{\gtrless k, 0}(\mathrm{X} B)$ from the right and the second one retrieved from Eq. (15) by setting $\mathrm{X} A=\mathrm{K} B, n=2 k$, and $\omega=0$, and by the multiplication of the resulting expression by $\widehat{\mathcal{R}}_{n, \omega}(\mathbb{X} A)$ from the left. Applying Eq. (27) to the sum of the terms Id and VII gives

$$
\begin{aligned}
& \mathrm{Id}+\mathrm{VII}=\left\langle\widehat{\mathcal{R}}_{1, \omega^{*}}(Y) \widehat{\mathcal{P}}_{2}(W)+\widehat{\mathcal{P}}_{1}(Y) \widehat{\mathcal{R}}_{2,0}(W) \mid \widehat{\mathcal{R}}_{3, \omega}(Z)\right\rangle= \\
= & -\left\langle\widehat{\mathcal{R}}_{1, \omega^{*}}(Y) \widehat{\mathcal{R}}_{2,0}(W) \mid\left[F, \widehat{\mathcal{R}}_{3, \omega}(Z)\right]\right\rangle-\omega\left\langle\widehat{\mathcal{R}}_{1, \omega^{*}}(Y) \widehat{\mathcal{R}}_{2,0}(W) \mid \widehat{\mathcal{R}}_{3, \omega}(Z)\right\rangle .
\end{aligned}
$$

In view of Eq. (15) the commutator in Eq. (28) can be replaced by $-\omega \widehat{\mathcal{R}}_{3, \omega}(Z)-\widehat{\mathcal{P}}_{3}(Z)$. As a result the terms multiplied by $\omega$ cancel each other and finally the following expression is obtained

$$
\mathrm{Id}+\mathrm{VII}=\left\langle\widehat{\mathcal{R}}_{1, \omega^{*}}(Y) \widehat{\mathcal{R}}_{2,0}(W) \mid Z\right\rangle=\left\langle\Xi_{1}^{(0)}\left(\omega^{*}\right) T_{2}^{(1)} \mid Z\right\rangle,
$$

where in the last step the definitions of the $\Xi_{1}^{(0)}\left(\omega^{*}\right)$ and $T_{2}^{(1)}$ operators have been used.

In this way the first triple excitation operator has been eliminated. After applying Eq. (23) the formula (29) separates into four fragments

$$
\begin{gathered}
\mathrm{Id}+\mathrm{VII}=\left\langle\Xi_{1}^{(0)}\left(\omega^{*}\right) T_{2}^{(1)} \mid\left[X, T_{3}^{(2)}\right]\right\rangle+\left\langle\Xi_{1}^{(0)}\left(\omega^{*}\right) T_{2}^{(1)} \mid\left[W, \Omega_{2}^{(1)}(\omega)\right]\right\rangle+ \\
+\left\langle\Xi_{1}^{(0)}\left(\omega^{*}\right) T_{2}^{(1)} \mid\left[\left[W, T_{2}^{(1)}\right], \Omega_{1}^{(0)}(\omega)\right]\right\rangle+\frac{1}{2}\left\langle\Xi_{1}^{(0)}\left(\omega^{*}\right) T_{2}^{(1)} \mid\left[\left[X, T_{2}^{(1)}\right], T_{2}^{(1)}\right]\right\rangle .
\end{gathered}
$$

The last three terms of Eq. (30) already have the desired form, i.e. they do not contain any triple excitation operators. The diagrammatic analysis of possible con- 


\section{Molecular Physics}

tractions allows to rewrite the first part of the r.h.s. of this equationEq. (30) as a sum of two fragments

$$
\begin{aligned}
& \left\langle\Xi_{1}^{(0)}\left(\omega^{*}\right) T_{2}^{(1)} \mid\left[X, T_{3}^{(2)}\right]\right\rangle= \\
& =\left\langle\widehat{\mathcal{P}}_{1}\left(X \Xi_{1}^{(0)}\left(\omega^{*}\right)\right) T_{2}^{(1)} \mid T_{3}^{(2)}\right\rangle+\left\langle\Xi_{1}^{(0)}\left(\omega^{*}\right) \widehat{\mathcal{P}}_{2}\left(\left[X, T_{2}^{(1)}\right]\right) \mid T_{3}^{(2)}\right\rangle
\end{aligned}
$$

where we use the fact that the commutator $\left[X, T_{3}^{(2)}\right]$ can be replaced by the operator product $X T_{3}^{(2)}$ in this case, and that only hole-hole and particle-particle parts of the $X$ operator contribute to Eq. (31).

Let us now examine the term Ia. A sequence of transformations listed below changes this term to the form suitable for our purposes

$$
\begin{aligned}
& \mathrm{Ia}=\left\langle Y \mid \widehat{\mathcal{R}}_{1, \omega}\left(\left[X, T_{1}^{(3 t)}\right]\right)\right\rangle=\left\langle\widehat{\mathcal{R}}_{1, \omega^{*}}(Y) \mid\left[X, T_{1}^{(3 t)}\right]\right\rangle= \\
= & \left\langle\Xi_{1}^{(0)}\left(\omega^{*}\right) \mid X T_{1}^{(3 t)}\right\rangle=\left\langle\Xi_{1}^{(0)}\left(\omega^{*}\right) \mid X \widehat{\mathcal{R}}_{1,0}\left(\left[W, T_{3}^{(2)}\right]\right)\right\rangle= \\
= & \left\langle X \Xi_{1}^{(0)}\left(\omega^{*}\right) \mid \widehat{\mathcal{R}}_{1,0}\left(\left[W, T_{3}^{(2)}\right]\right)\right\rangle=\left\langle\widehat{\mathcal{R}}_{1,0}\left(X \Xi_{1}^{(0)}\left(\omega^{*}\right)\right) \mid\left[W, T_{3}^{(2)}\right]\right\rangle= \\
= & \left\langle\widehat{\mathcal{R}}_{1,0}\left(X \Xi_{1}^{(0)}\left(\omega^{*}\right)\right) \widehat{\mathcal{P}}_{2}(W) \mid T_{3}^{(2)}\right\rangle .
\end{aligned}
$$

If the result of derivations presented in Eq. (32) and the first term of the r.h.s. of Eq. (31) are summed up, the following expression results

$$
\begin{aligned}
& \left\langle\widehat{\mathcal{P}}_{1}\left(X \Xi_{1}^{(0)}\left(\omega^{*}\right)\right) T_{2}^{(1)} \mid T_{3}^{(2)}\right\rangle+\left\langle\widehat{\mathcal{R}}_{1,0}\left(X \Xi_{1}^{(0)}\left(\omega^{*}\right)\right) \widehat{\mathcal{P}}_{2}(W) \mid T_{3}^{(2)}\right\rangle= \\
= & \left\langle\widehat{\mathcal{R}}_{1,0}\left(X \Xi_{1}^{(0)}\left(\omega^{*}\right)\right) T_{2}^{(1)} \mid\left[W, T_{2}^{(1)}\right]\right\rangle .
\end{aligned}
$$

In the derivation of Eq. (33) the identity [33]

$$
\widehat{\mathcal{P}}_{k}(\mathrm{~K} B) \widehat{\mathcal{R}}_{n, \omega}(\mathrm{X} A)+\widehat{\mathcal{R}}_{k,-\omega}(\mathrm{K} B) \widehat{\mathcal{P}}_{n}(\mathrm{X} A)+\left[F, \widehat{\mathcal{R}}_{k,-\omega}(\mathrm{K} B) \widehat{\mathcal{R}}_{n, \omega}(\mathrm{X} A)\right]=0,
$$

has been employed for $\omega=0, k=1$, and $n=2$. Eq. (34) ishas been obtained by using the same procedure as for the case of Eq. (27). Eq. (33) does not contain any triple excitation operators, but can be still somewhat simplified. If the operator $T_{2}^{(1)}$ is moved to the ket side and the Hermicity of the $\widehat{\mathcal{R}}_{n, 0}$ superoperator is used, then the r.h.s. of Eq. (33) can be rewritten as $\left\langle X \Xi_{1}^{(0)}\left(\omega^{*}\right) \mid \Sigma_{1}^{(3)}\right\rangle$, where the auxiliary operator $\Sigma_{1}^{(3)}$ is defined as

$$
\Sigma_{1}^{(3)}=\widehat{\mathcal{R}}_{1,0}\left(T_{2}^{(1) \dagger}\left[W, T_{2}^{(1)}\right]\right)
$$

Note that the $\Sigma_{1}^{(3)}$ operator is identical with the second term of the $\Lambda_{1}^{(3)}$ operator (see Eq. (19)). This part of the derivation is represented diagrammatically in the Appendix.

Finally, it can be found from the examination of Table 3 that the counterpart (in a sense of Eq. (34)) for the second fragment from the r.h.s. of Eq. (31) is the g.c.c. of the term IVa, which can be rewritten as

$$
\text { g.c.c.IVa }=\left\langle\widehat{\mathcal{R}}_{2,-\omega^{*}}\left(\left[X, T_{2}^{(1)}\right]\right) \mid\left[Y, T_{3}^{(2)}\right]\right\rangle=\left\langle\widehat{\mathcal{R}}_{2,-\omega^{*}}\left(\left[X, T_{2}^{(1)}\right]\right) \widehat{\mathcal{P}}_{1}(Y) \mid T_{3}^{(2)}\right\rangle .
$$

The sum of these two terms gives $\left\langle\widehat{\mathcal{R}}_{2,-\omega^{*}}\left(\left[X, T_{2}^{(1)}\right]\right) \Xi_{1}^{(0)}\left(\omega^{*}\right) \mid\left[W, T_{2}^{(1)}\right]\right\rangle$. 


\subsubsection{Terms II and III}

The techniques presented in Section 3.2.1 can be applied to the terms II and III giving

$$
\begin{aligned}
\mathrm{II} & =\left\langle T_{1}^{(3 t)} \mid Y \Omega_{1}^{(0)}(\omega)\right\rangle=\left\langle\widehat{\mathcal{R}}_{1,0}\left(\left[W, T_{3}^{(2)}\right]\right) \mid Y \Omega_{1}^{(0)}(\omega)\right\rangle= \\
& =\left\langle\left[W, T_{3}^{(2)}\right] \mid \widehat{\mathcal{R}}_{1,0}\left(Y \Omega_{1}^{(0)}(\omega)\right)\right\rangle=\left\langle T_{3}^{(2)} \mid \widehat{\mathcal{P}}_{2}(W) \widehat{\mathcal{R}}_{1,0}\left(Y \Omega_{1}^{(0)}(\omega)\right)\right\rangle
\end{aligned}
$$

and

$$
\begin{aligned}
\mathrm{III} & =\left\langle\left[Y, \widehat{\mathcal{P}}_{1}\left(\left[T_{2}^{(1) \dagger}, T_{3}^{(2)}\right]\right)\right] \mid \Omega_{1}^{(0)}(\omega)\right\rangle=\left\langle T_{2}^{(1) \dagger} T_{3}^{(2)} \mid \widehat{\mathcal{P}}_{1}\left(Y \Omega_{1}^{(0)}(\omega)\right)\right\rangle= \\
& =\left\langle T_{3}^{(2)} \mid T_{2}^{(1)} \widehat{\mathcal{P}}_{1}\left(Y \Omega_{1}^{(0)}(\omega)\right)\right\rangle=\left\langle T_{3}^{(2)} \mid \widehat{\mathcal{R}}_{2,0}(W) \widehat{\mathcal{P}}_{1}\left(Y \Omega_{1}^{(0)}(\omega)\right)\right\rangle .
\end{aligned}
$$

As before, by applying a sequence of Eqs. (34) and (15) the sum of these two terms can be transformed to the following expression

$$
\mathrm{II}+\mathrm{III}=\left\langle\left[W, T_{2}^{(1)}\right] \mid T_{2}^{(1)} \widehat{\mathcal{R}}_{1,0}\left(Y \Omega_{1}^{(0)}(\omega)\right)\right\rangle .
$$

Finally, if the $T_{2}^{(1)}$ operator in Eq. (39) is moved to the bra side and the definition of the operator $\Sigma_{1}^{(3)}$ is again used (see Eq. (35)), then the final form of Eq. (39) can be written as

$$
\mathrm{II}+\mathrm{III}=\left\langle\Sigma_{1}^{(3)} \mid Y \Omega_{1}^{(0)}(\omega)\right\rangle
$$

\subsubsection{Terms Ib, Ic, IVb, V}

The summation of the term $\mathrm{V}$

$$
\mathrm{V}=\left\langle\left[Y, T_{2}^{(3 t)}\right] \mid \Omega_{1}^{(0)}(\omega)\right\rangle=\left\langle T_{2}^{(3 t)} \mid \widehat{\mathcal{P}}_{1}(Y) \Omega_{1}^{(0)}(\omega)\right\rangle
$$

and of the g.c.c. of the term Ib

$$
\begin{aligned}
& \text { g.c.c. } \mathrm{Ib}=\left\langle\widehat{\mathcal{R}}_{1, \omega^{*}}\left(\left[X, T_{2}^{(3 t)}\right]\right) \mid Y\right\rangle=\left\langle\left[X, T_{2}^{(3 t)}\right] \mid \Xi_{1}^{(0)}(-\omega)\right\rangle= \\
= & \left\langle T_{2}^{(3 t)} \mid \widehat{\mathcal{P}}_{1}(X) \Xi_{1}^{(0)}(-\omega)\right\rangle,
\end{aligned}
$$

results in the following expression

$$
\text { g.c.c. } \mathrm{Ib}+\mathrm{V}=\left\langle\left[W, T_{3}^{(2)}\right] \mid \Omega_{1}^{(0)}(\omega) \Xi_{1}^{(0)}(-\omega)\right\rangle .
$$

WhenIf the operator $W$ is moved to the ket side and the diagrammatic analysis of possible contractions is performed, the following two terms are obtained

$$
\begin{aligned}
& \text { g.c.c. } \mathrm{Ib}+\mathrm{V}= \\
= & \left\langle T_{3}^{(2)} \mid \widehat{\mathcal{P}}_{2}\left(W \Omega_{1}^{(0)}(\omega)\right) \Xi_{1}^{(0)}(-\omega)\right\rangle+\left\langle T_{3}^{(2)} \mid \Omega_{1}^{(0)}(\omega) \widehat{\mathcal{P}}_{2}\left(W \Xi_{1}^{(0)}(-\omega)\right)\right\rangle .
\end{aligned}
$$

The first of the terms on the r.h.s. of Eq. (44) can be paired with the term IVb,

$$
\mathrm{IVb}=\left\langle\left[Y, T_{3}^{(2)}\right] \mid \widehat{\mathcal{R}}_{2, \omega}\left(\left[W, \Omega_{1}^{(0)}(\omega)\right]\right)\right\rangle=\left\langle T_{3}^{(2)} \mid \widehat{\mathcal{R}}_{2, \omega}\left(\left[W, \Omega_{1}^{(0)}(\omega)\right]\right) \widehat{\mathcal{P}}_{1}(Y)\right\rangle
$$


while the second term of Eq. (44) makes a pair with the g.c.c. of the term Ic-

$$
\begin{aligned}
& \text { g.c.c.Ic }=\left\langle\widehat{\mathcal{R}}_{1,-\omega^{*}}\left(\left[W, \Omega_{2}^{(2 t)}\left(-\omega^{*}\right)\right]\right) \mid Y\right\rangle=\left\langle\left[W, \Omega_{2}^{(2 t)}\left(-\omega^{*}\right)\right] \mid \Xi_{1}^{(0)}(-\omega)\right\rangle= \\
= & \left\langle\Omega_{2}^{(2 t)}\left(-\omega^{*}\right) \mid \widehat{\mathcal{P}}_{2}\left(W \Xi_{1}^{(0)}(-\omega)\right)\right\rangle=\left\langle T_{3}^{(2)} \mid \widehat{\mathcal{P}}_{1}(X) \widehat{\mathcal{P}}_{2} \widehat{\mathcal{R}}_{2,-\omega}\left(W \Xi_{1}^{(0)}(-\omega)\right)\right\rangle,
\end{aligned}
$$

respectively.

Summarizing, the final result of this Section is listed below

$$
\begin{aligned}
\text { g.c.c.Ib }+ \text { g.c.c.Ic }+\mathrm{IVb}+\mathrm{V} & =\left\langle\left[W, T_{2}^{(1)}\right] \mid \Omega_{1}^{(0)}(\omega) \widehat{\mathcal{R}}_{2,-\omega}\left(W \Xi_{1}^{(0)}(-\omega)\right)\right\rangle+ \\
& +\left\langle\left[W, T_{2}^{(1)}\right] \mid \widehat{\mathcal{R}}_{2, \omega}\left(W \Omega_{1}^{(0)}(\omega)\right) \Xi_{1}^{(0)}(-\omega)\right\rangle= \\
=\left\langle\left[W, T_{2}^{(1)}\right] \mid \widehat{\mathcal{R}}_{2, \omega}\left(W \Omega_{1}^{(0)}(\omega)\right) \Xi_{1}^{(0)}(-\omega)\right\rangle & + \text { op.ex. }
\end{aligned}
$$

\subsubsection{Terms VI and VIII}

The remaining components in Table 3 are the terms VI and VIII. The g.c.c. of the term VI can be rewritten as

$$
\begin{aligned}
& \text { g.c.c. } \mathrm{VI}=\left\langle\Omega_{2}^{(2 t)}\left(-\omega^{*}\right) \mid\left[Y, T_{2}^{(1)}\right]\right\rangle=\left\langle\widehat{\mathcal{R}}_{2,-\omega^{*}}\left(\left[X, T_{3}^{(2)}\right]\right) \mid\left[Y, T_{2}^{(1)}\right]\right\rangle= \\
& =\left\langle T_{3}^{(2)} \mid \widehat{\mathcal{P}}_{1}(X) \widehat{\mathcal{R}}_{2,-\omega}\left(\left[Y, T_{2}^{(1)}\right]\right)\right\rangle .
\end{aligned}
$$

and similarly the term VIII can be transformed to

$$
\mathrm{VIII}=\left\langle T_{3}^{(2)} \mid \Omega_{1}^{(0)}(\omega) \widehat{\mathcal{P}}_{2}\left(\left[Y, T_{2}^{(1)}\right]\right)\right\rangle
$$

in a few steps explained in detail in the previous sections. It is easy to see that the summation of these two terms leads to the result

$$
\text { g.c.c. VI }+\mathrm{VIII}=\left\langle\left[W, T_{2}^{(1)}\right] \mid \Omega_{1}^{(0)}(\omega) \widehat{\mathcal{R}}_{2,-\omega}\left(\left[Y, T_{2}^{(1)}\right]\right)\right\rangle,
$$

what completes the derivation of the correction terms for the XCCSD polarization propagator.

\subsubsection{The final correction formula for the XCCSD polarization propagator}

When the partial results from Sections 3.2.1-3.2.4 are collected together and the definitions of the $\Omega_{2}^{(1)}(\omega)$ and $\Xi_{2}^{(1)}(-\omega)$ operators from Eq. (16) are utilized, then the final correction formula for the triple contributions of the third MP order can be written as

$$
\begin{aligned}
& \Delta^{(3 t)}(\mathrm{XCC})=\left\langle\Xi_{1}^{(0)}\left(\omega^{*}\right) T_{2}^{(1)} \mid\left[W, \Omega_{2}^{(1)}(\omega)\right]+\left[\left[W, T_{2}^{(1)}\right], \Omega_{1}^{(0)}(\omega)\right]+\frac{1}{2}\left[\left[X, T_{2}^{(1)}\right], T_{2}^{(1)}\right]\right\rangle+ \\
+ & \left\langle X \Xi_{1}^{(0)}\left(\omega^{*}\right) \mid \Sigma_{1}^{(3)}\right\rangle+\left\langle\Sigma_{1}^{(3)} \mid Y \Omega_{1}^{(0)}(\omega)\right\rangle+ \\
+ & \left\langle\left[W, T_{2}^{(1)}\right] \mid \Omega_{2}^{(1)}(\omega) \Xi_{1}^{(0)}(-\omega)\right\rangle+\left\langle\left[W, T_{2}^{(1)}\right] \mid \Omega_{1}^{(0)}(\omega) \Xi_{2}^{(1)}(-\omega)\right\rangle+\text { g.c.c., }
\end{aligned}
$$

where g.c.c. denotes, as usual, that for each term its g.c.c. counterpart should be added in order to obtain the complete formula: note that in the derivations presented in Sections 3.2.1-3.2.4 either the term $k$, or the g.c.c. of the term $k$ (but never both terms simultaneously) has been employed. Eq. (51) includes the lowest nonvanishing MP orders of the $T, \Omega$, and $\Xi$ operators. It can be therefore proposed 
to replace the MP operators by their converged CCSD counterparts, what results in the following formula,

$$
\begin{aligned}
& \Delta(\mathrm{XCC})=\left\langle\Xi_{1}\left(\omega^{*}\right) T_{2} \mid\left[W, \Omega_{2}(\omega)\right]+\left[\left[W, T_{2}\right], \Omega_{1}(\omega)\right]+\frac{1}{2}\left[\left[X, T_{2}\right], T_{2}\right]\right\rangle+ \\
+ & \left\langle\Sigma_{1} \mid Y \Omega_{1}(\omega)\right\rangle+\left\langle X \Xi_{1}\left(\omega^{*}\right) \mid \Sigma_{1}\right\rangle+ \\
+ & \left\langle\left[W, T_{2}\right] \mid \Omega_{2}(\omega) \Xi_{1}(-\omega)\right\rangle+\left\langle\left[W, T_{2}\right] \mid \Omega_{1}(\omega) \Xi_{2}(-\omega)\right\rangle+\text { g.c.c. }
\end{aligned}
$$

where the $\Sigma_{1}$ operator is defined as

$$
\left[F, \Sigma_{1}\right]+\widehat{\mathcal{P}}_{1}\left(T_{2}^{\dagger}\left[W, T_{2}\right]\right)=0
$$

Eq. (52) is the final result of this section. It includes all $\mathcal{O}\left(W^{3}\right)$ contributions of the XCC polarization propagator, but does not contain triple excitation operators and can be calculated forat the $\mathcal{O}\left(\mathcal{N}^{6}\right)$ cost (a more detailed analysis reveals that the most expensive parts of this formula scale as $\mathcal{O}\left(o^{3} v^{3}\right)$, where $o$ and $v$ are dimensions of occupied and virtual orbital spaces, respectively). If the correction $\Delta(\mathrm{XCC})$ is added to the XCCSD polarization propagator, a low-cost propagator correct through the third MP order is obtained.

\subsection{The elimination of triple excitation operators from the MP3 contributions of the TD-CC polarization propagator}

A similar correction formula can be proposed for the TD-CCSD polarization propagator. The MP3 terms missing in the TD-CCSD formula are collected in Table 4. Since they are identical to some terms from Table 3, the same roman labels are preserved. It is important to mention that the component 3 from Table 1 does not contribute to Table 4 , because the operator $\Lambda_{1}^{(3 t)}$ is equal to zero, while a similar component 3 from Table 2 gives rise to the terms II and III of Table 3.

An identification of the correction terms for the TD-CCSD case is, however, not as straightforward, as it appears from the first look at Table 4, since for each of listed contributions its op.ex. counterpart instead of the g.c.c. counterpart should be added. Since in the derivations presented in Sections 3.2.1-3.2.4 the g.c.c. of some terms from Table 3 have been used in several places, these cases should be reexamined once more in order to check if the new appropriate pairing terms can be found.

\subsubsection{Terms Ia, Ib, Ic, Id, VI, and VII for the TD-CCSD case}

Let us first analyse the terms from the first line of Table 4, which have appeared in Sections 3.2.1 and 3.2.3. In Section 3.2.1 the g.c.c. of the term IVa (Eq. (36)) is utilized as the counterpart of one of the terms arising during the derivation. A closer look at Table 4 reveals that this term is identical to the op.ex. of the term VI. Since all other terms from Section 3.2.1 are explicitly listed in Table 4, the final result of this section is valid also for the TD-CC case. The remaining terms from Table 4 are the terms Ib and Ic, while in Section 3.2.3 the following terms: the g.c.c. of the term Ib, the g.c.c. of the term Ic, IVb, and $\mathrm{V}$ have been considered. Alternatively, one could make the derivations from Section 3.2.3 for the g.c.c. of the abovementioned terms, i.e. for the terms Ib, Ic, the g.c.c. of IVb, and the g.c.c. of $\mathrm{V}$ (they are included in Eq. (51) anyway under the abbreviation g.c.c.). After some operator manipulations it can be shown that the g.c.c. of IVb is identical to the op.ex. of Ic and the g.c.c. of $\mathrm{V}$ is equal to the op.ex. of Ib, so the g.c.c. of the final result of Section 3.2.3 can be used also for the TD-CCSD triples correction. 
It should be stressed that in this case both terms have been utilized together with their op.ex. counterparts, so the g.c.c. of the r.h.s. of Eq. (47) should appear only once in the final formula.

\subsubsection{The final correction formula for the TD-CCSD polarization propagator}

After collecting the results from Sections 3.2.1 and 3.2.3 the following triples correction for the TD-CC polarization propagator can be proposed

$$
\begin{aligned}
\Delta^{(3 t)}(\mathrm{TD}) & =\left\langle\Xi_{1}^{(0)}\left(\omega^{*}\right) T_{2}^{(1)} \mid\left[W, \Omega_{2}^{(1)}(\omega)\right]+\left[\left[W, T_{2}^{(1)}\right], \Omega_{1}^{(0)}(\omega)\right]+\frac{1}{2}\left[\left[X, T_{2}^{(1)}\right], T_{2}^{(1)}\right]\right\rangle+ \\
& +\left\langle X \Xi_{1}^{(0)}\left(\omega^{*}\right) \mid \Sigma_{1}^{(3)}\right\rangle+\left\langle\Omega_{2}^{(1)}\left(-\omega^{*}\right) \Xi_{1}^{(0)}\left(\omega^{*}\right) \mid\left[W, T_{2}^{(1)}\right]\right\rangle+\text { op.ex. }
\end{aligned}
$$

where op.ex. denotes that for each term its op.ex. counterpart should be added in order to restore the complete formula. After replacing the MP operators by their CCSD counterparts the following expression is obtained for the triple-excitation correction for the TD-CCSD case,

$$
\begin{aligned}
\Delta(\mathrm{TD}) & =\left\langle\Xi_{1}\left(\omega^{*}\right) T_{2} \mid\left[W, \Omega_{2}(\omega)\right]+\left[\left[W, T_{2}\right], \Omega_{1}(\omega)\right]+\frac{1}{2}\left[\left[X, T_{2}\right], T_{2}\right]\right\rangle+ \\
& +\left\langle X \Xi_{1}\left(\omega^{*}\right) \mid \Sigma_{1}\right\rangle+\left\langle\Omega_{2}\left(-\omega^{*}\right) \Xi_{1}\left(\omega^{*}\right) \mid\left[W, T_{2}\right]\right\rangle+\text { op.ex. }
\end{aligned}
$$

Eq. (55) is the main result of this section. Similarly as for the XCCSD case, if the correction $\Delta(\mathrm{TD})$ is added to the TD-CCSD propagator, the expression accurate through the $\mathcal{O}\left(W^{3}\right)$ order is obtained at the $\mathcal{O}\left(\mathcal{N}^{6}\right)$ computational cost.

\subsection{A comparison of the XCCSD and TD-CCSD correction formulae}

The formulae (51) and (54) (or alternatively (52) and (55)) are similar, but not identical, e.g. it is evident that the second equation contains less terms than the first one. In particular, one term with the $\Sigma_{1}^{(3)}$ operator and one term containing the product of the first-order single and double excitation operators are absent in the second expression. In order to explain this fact let us first analyse triples contributions to the $S_{1}^{(3)}$ operator (see Eq. (21)). One of the terms from Eq. (21) originates from the $T_{1}^{(3)}$ operator, while the second one - from the $T^{\dagger} T$ part of the $S$ operator. The sum of these two terms projected on some arbitrary single excitation operator $\tau_{1}$ gives

$$
\begin{aligned}
& \left\langle\tau_{1} \mid \widehat{\mathcal{R}}_{1,0}\left(W T_{3}^{(2)}\right)\right\rangle+\left\langle\tau_{1} \mid \widehat{\mathcal{P}}_{1}\left(\left(T_{2}^{(1)}\right)^{\dagger} T_{3}^{(2)}\right)\right\rangle= \\
= & \left\langle\widehat{\mathcal{R}}_{1,0}\left(\tau_{1}\right) \widehat{\mathcal{P}}_{2}(W) \mid T_{3}^{(2)}\right\rangle+\left\langle\tau_{1} \widehat{\mathcal{R}}_{2,0}(W) \mid T_{3}^{(2)}\right\rangle=\left\langle\widehat{\mathcal{R}}_{1,0}\left(\tau_{1}\right) T_{2}^{(1)} \mid\left[W, T_{2}^{(1)}\right]\right\rangle,
\end{aligned}
$$

where once more the sequence of Eqs. (34) and (15) have been employed. The result of these derivations is identical with the second term of Eq. (19) for the $\Lambda_{1}^{(3)}$ operator. It is straightforward to see that also other parts of the operators $\Lambda_{1}^{(3)}$ and $S_{1}^{(3)}$ are the same, so finally one can write $\Lambda_{1}^{(3)}=S_{1}^{(3)}$. However, if the XCCSD polarization propagator is calculated (the $\operatorname{CCSD}(3)$ model from Ref. [34]), an approximate form of $S_{1}$ is utilized, which is constructed from the $T_{1}$ and $T_{2}$ operators of CCSD theory. The components absent for the CCSD case (see Eq. (56)) give rise to the terms II and III from Table 3 and are responsible for one contribution containing $\Sigma_{1}^{(3)}$ in Eq. (51). The second "missing" term in Eq. (55) can be identified as the term 10 from Table 1. After some manipulations it can be demonstrated that this 
term can be rewritten as $\left\langle\left[W, \Lambda_{2}\right] \mid \Omega_{1}(\omega) \Xi_{2}(-\omega)\right\rangle$, i.e. it represents exactly the last term of Eq. (52).

\section{Summary}

The analysis of the most important contributions for the TD-CC and XCC polarization propagators has been performed by means of MP theory. The TD-CCSD and XCCSD polarization propagators, which in their original formulations are rigorously correct through the second order of MP theory only, have been augmented with noniterative corrections $\Delta(\mathrm{XCC})$ and $\Delta(\mathrm{TD})$, respectively, accounting for the missing triple contributions on the MP3 level. In the final formulae triple excitation operators have been eliminated by utilizing techniques which enable the removal of long energy denominators on the operator level. Since missing triple contributions of the leading MP3 order are responsible for a less satisfactory performance of the TD-CCSD and XCCSD polarization propagators as compared to the CCSD correlation energy [which is rigorously correct through the $\mathcal{O}\left(W^{3}\right)$ order], it can be expected that the proposed correction terms will improve the agreement between the CCSD polarization propagators and the full configuration interaction (FCI) benchmarks. The scaling of the computational time for Eqs. (52) and (55) is at least one order of magnitude lower than for the case of an explicit use of triple excitation operators (e.g. the computational time for the TD-CC3 polarization propagator scales as iterative $\mathcal{O}\left(o^{3} v^{4}\right)[19]$, while the proposed corrections can be obtained with the noniterative $\mathcal{O}\left(o^{3} v^{3}\right)$ cost). The low cost of the $\Delta(\mathrm{XCC})$ and $\Delta(\mathrm{TD})$ corrections will allow to perform calculations for CC polarization propagators accurate through the $\mathcal{O}\left(W^{3}\right)$ order for much larger molecules and orbital basis sets than it is possible with e.g. the TD-CC3 method. The comparison of the XCC and TD-CC approaches on the operator level reveals that that some terms, which appear in the XCC model only when triple excitations are included, are already incorporated in the original TD-CCSD polarization propagator, either with help of the double commutator term $\left\langle\Lambda_{2} \mid\left[\left[\bar{H}, \Omega_{1}(\omega)\right], \Xi_{2}(-\omega)\right]\right\rangle$, or through the $\Lambda_{1}$ operator. However, numerical tests show that errors of both methods with respect to the FCI benchmarks are of the same order of magnitude, although on average they are somewhat smaller for the TD-CCSD case [34]. The implementation of Eqs. (52) and (55) is planned in the near future.

\section{Appendix: On the use of diagrams for the derivation of the $\Delta$ (XCC) correction}

The derivations presented in Sections 3.2.1-3.2.4 can be greatly simplified if the diagrammatic approach is utilized as an auxiliary tool. For this purpose it is enough to employ the arrowless (nonoriented) Hugenholtz diagrams [46]. In the following it will be demonstrated on one selected example from Section 3.2.1 how one can utilize diagrams in order to find the counterparts of some term under study. The following drawing conventions will be used for operator vertices: a small filled circle denotes the $W$ operator, an empty (white) circle - the $T$ operator, a cross - the $X$ operator, a crossedsmall circle with the letter $Y$ inside - the $Y$ operator, a black square the $\Omega$ operator and finally a large filled circle - the $\Xi$ operator. Thin vVertical lines denote energy denominators from Eq. (14), with a corresponding frequency listed above or below the denominator line. Additionally, auxiliary operators, like the $W T_{n}$ operators or the $Z$ operator defined in Eq. (23) are represented by an ellipse with the corresponding symbol inside. A left-to-right drawing convention is 
used, i.e. for a formula $\langle A B C \ldots\rangle$ the order of vertices $A, B, C$, etc. corresponds to their order in the formula. As usual, if operators commute the order of their vertices in a diagram can be changed and e.g. these vertices can be drawn one over another, if convenient.

Let us reiterate the search for the complementary terms, which has been the main issue in Sections 3.2.1 to 3.2.4, by utilizing the diagrammatic methods.

tet us now take as an example the first term of the r.h.s. of Eq. (31). This term is shown on Figure $1(a)$ with the $\Xi_{1}^{(0)}\left(\omega^{*}\right)$ and $T_{2}^{(1)}$ vertices and on Figure $1(b)$ with the $T_{2}^{(1)}$ vertex replaced by $\widehat{\mathcal{R}}_{2,0}(W)$ and $\Xi_{1}^{(0)}\left(\omega^{*}\right)$ replaced by $\widehat{\mathcal{R}}_{1, \omega^{*}}(Y)$. From Eq. (34) it follows that the appropriate "pairing" counterpart for this term has the reverse pattern of the denominator lines, like depicted on Figure 2(a). It is easy to see that the diagram from Figure 2(a) includes the $T_{1}^{(3 t)}$ operator, presented alone on Figure 2(b). This in turn allows to identify the whole diagram from Figure $\mathcal{Z}(a)$ as $\left\langle Y \mid \widehat{\mathcal{R}}_{1, \omega}\left(X T_{1}^{(3 t)}\right)\right\rangle$. This term represents exactly the term Ia from Table 3. In this way the counterpart of the first term of the r.h.s. of Eq. (31) has been identified. Other cases from Sections $3.2 .1-3.2 .4 \mathrm{can}$ be proceeded in a similar way.

The first term examined in Section 3.2.1 is the term Id (see Eq. (24)), which is shown in Figure 1(a). Its rightmost energy denominator can be eliminated, if this diagram is considered together with a diagram presented in Figure 1(b), having the reverse pattern of the short (i.e. corresponding to single or double excitations) denominator lines. The frequency in the latter diagram (i.e. zero) has been obtained from the condition that the total frequency should be equal to the frequency in the long energy denominator. In the diagram shown in Figure 1(b) the Hermitian adjoint of the doubly-excited cluster operator $T_{2}^{(1)}$ can be easily seen (see Figure $1(\mathrm{c})$ ), while the remaining part of the diagram contains the $Y$ operator contracted to the $\Omega_{3}^{(2)}(\omega)$ operator, so that finally this diagram can be identified as the term VII. The sum of both terms gives the term depicted in Figure 1(d), i.e. it represents the diagram where both short energy denominator lines are present, but the troublesome long line vanishes. The same result on the operator level has been obtained in a sequence of Eqs. (24)-(29). In order to clarify the correspondence between the diagrammatic and algebraic approaches, let us write explicitly the algebraic expression obtained from the diagram presented in Figure 1(a). For this purpose this diagram has been decorated with arrows and line labels (indices labelling the occupied and virtual spinorbitals are denoted by letters $i, j, k$ and $a, b, c$, respectively). The algebraic form of the diagram from Figure 1(a) (with amplitudes $Y_{i}^{a}$ etc. implicitly defined) is given below

$$
\mathrm{Id}=\sum_{i j k a b c} Y_{i}^{a}\left(\epsilon_{i}-\epsilon_{a}-\omega\right)^{-1} W_{j k}^{b c}\left(\epsilon_{i}+\epsilon_{j}+\epsilon_{k}-\epsilon_{a}-\epsilon_{b}-\epsilon_{c}-\omega\right)^{-1} Z_{a b c}^{i j k}
$$

It can be easily verified that the expression for the neighbour diagram (see Figure 1(b)) differs only by the absence of the term $\left(\epsilon_{i}-\epsilon_{a}-\omega\right)^{-1}$ and the presence of $\left(\epsilon_{j}+\epsilon_{k}-\epsilon_{b}-\epsilon_{c}\right)^{-1}$. The rest of the procedure for the elimination of the triple energy denominator has been described at the beginning of Section 3.2.

In the following the diagrams containing the $T_{3}^{(2)}$ operator will be examined in detail. For clarity reasons the full diagrammatic representation of this operator (with the reduced resolvent $\widehat{\mathcal{R}}_{3,0}$ and the $\left[W, T_{2}^{(1)}\right]$ commutator, cf. Figure 2(a)) will be not used here. Instead, either a simple $T$ vertex for $T_{3}^{(2)}$, cf. Figure 2(b), or a simplified version of diagram shown in Figure 2(b), as given in Figure 2(c), will be utilized. 
The next identity which can be clarified by the diagrammatic approach is the result presented in Eq. (31). Since in the 1.h.s. of this equation the one-electron operator $X$ should be connected to the $T_{3}^{(2)}$ vertex (because of the presence of the commutator with $T_{3}^{(2)}$ ), the remaining line of the $X$ vertex connects either to the $\Xi_{1}^{(0)}\left(\omega^{*}\right)$, or to the $T_{2}^{(1)}$ vertex, giving two terms presented in Figure 3.

The first term of the r.h.s. of Eq. (31) is shown in Figure $4(\mathrm{a})$ with the $T_{2}^{(1)}$ vertex replaced by $\widehat{\mathcal{R}}_{2,0}(W)$ and $\Xi_{1}^{(0)}\left(\omega^{*}\right)-$ by $\widehat{\mathcal{R}}_{1, \omega^{*}}(Y)$. The complementary term should have the reverse pattern of the energy denominator lines, as depicted in Figure 4(b). It can be observed that the diagram from Figure 4(b) includes the $T_{1}^{(3 t)}$ operator, presented separately in Figure 4(c). The remaining elements of the diagrams (from the left to the right) are: $Y, \widehat{\mathcal{R}}_{1, \omega}$, and $X$, so finally the diagram from Figure $4(\mathrm{~b})$ can be identified as $\left\langle Y \mid \widehat{\mathcal{R}}_{1, \omega}\left(X T_{1}^{(3 t)}\right)\right\rangle$, i.e. the term Ia. In this way the counterpart of the first term from the r.h.s. of Eq. (31) has been found. The sum of the diagrams shown in Figures 4(a) and 4(b) is presented by the diagram from Figure 4(d) (see also Eq. (33)).

We proceed similarly for the second term from the r.h.s. of Eq. (31). Its diagram is presented in Figure $5(\mathrm{a})$ with the $\Xi_{1}^{(0)}\left(\omega^{*}\right)$ operator replaced by $\widehat{\mathcal{R}}_{1, \omega^{*}}(Y)$, while the complementary diagram is depicted in Figure $5(\mathrm{~b})$. The latter diagram can be identified as g.c.c. of the term IVa. For the sake of brevity the final diagrams obtained from the summation of the diagram pairs will be not shown in the rest of this Appendix, since the way of the elimination of long energy denominators is strictly analogous to this presented in Figure 4.

In Section 3.2.2 a counterpart for the term II is looked for. The term II is shown in Figure 6(a) with the Hermitian adjoint of the $T_{1}^{(3 t)}$ operator explicitly inserted. Its complementary term with the reverse pattern of short energy denominators is presented in Figure 6(b). In this diagram one can recognize the $T_{2}^{(1)}$ operator connected to the Hermitian adjoint of the $T_{3}^{(2)}$ operator. Together these operators form a single deexcitation operator connected to the $Y$ and $\Omega_{1}^{(0)}(\omega)$ operators, so finally this diagram can be identified as the term III.

Sections 3.2.3 and 3.2.4 deal with terms containing the double excitation operators $T_{2}^{(3 t)}$ and $\Omega_{2}^{(2 t)}(\omega)$. These operators are presented in Figure 7 . For the sake of brevity the diagrammatic representation of Eqs. (41)-(43), showing the elimination of the double energy denominator of $T_{2}^{(3 t)}$ is skipped here and only the result of these derivations, already splitted into two possible diagrams, is given in Figures 8(a) and 8(c). The counterparts of these two diagrams are presented in Figures 8(b) and $8(\mathrm{~d})$, respectively. An examination of the diagram shown in Figure 8(c) reveals that the Hermitian adjoint of the $T_{3}^{(2)}$ operator is connected to the operators $Y$ and $\widehat{\mathcal{R}}_{2, \omega}\left(\left[W, \Omega_{1}^{(0)}(\omega)\right]\right)$, what allows to identify this term as IVb. From an analysis of the diagram presented in Figure $8(\mathrm{~d})$ one can see that the Hermitian adjoint of the $\Omega_{2}^{(2 t)}(\omega)$ operator (see Figure $7(\mathrm{~b})$ ) is present in this diagram, what identifies it as g.c.c. of the term Ic.

Finally, the term VIII, shown in Figure 9(a), has the counterpart with exchanged short denominator lines depicted in Figure 9(b). On the latter diagram the Hermitian adjoint of the $\Omega_{2}^{(2 t)}(\omega)$ operator can be again recognized. This in turn leads to the identification of this diagram as g.c.c. of the term VI, what finalizes the diagrammatic derivation of the $\Delta^{(3 t)}(\mathrm{XCC})$ correction term. 


\section{References}

[1]J. CizeK, J. Chem. Phys. 45, 4256 (1966).

[2] K. Raghavachari, G. W. Trucks, J. A. Pople, and M. Head-Gordon, Chem. Phys. Lett. 157, 479 (1989).

[3]R. J. Bartlett, J. Phys. Chem. 93, 1697 (1989).

[4] M. Nooijen, K. R. Shamasundar, and D. Mukherjee, Mol. Phys. 103, 2277 (2005).

[5]J. Paldus and X. Li, Adv. Chem. Phys. 110, 1 (1999).

[6]H. J. Monkhorst, Int. J. Quantum Chem. Symp. 11, 421 (1977).

[7]E. Dalgand and H. J. Monkhorst, Phys. Rev. A 28, 1217 (1983).

[8]L. Adamowicz, W. D. Laidig, and R. J. Bartlett, Int. J. Quantum Chem. Symp. 18, 245 (1984).

[9]R. J. BARtLet, Analytical evaluation of gradients in coupled cluster and many body perturbation theory, in Geometrical Derivatives of Energy Surfaces and Molecular Properties, edited by P. JøRGENSEN and J. Simons, p. 35, Reidel, Dordrecht, The Netherlands, 1986.

[10]E. A. Salter, G. W. Trucks, and R. J. Bartlett, J. Chem. Phys. 90, 1752 (1989).

[11] A. E. Kondo, P. Piecuch, and J. Paldus, J. Chem. Phys. 102, 6511 (1995).

[12]P. Jørgensen and T. Helgaker, J. Chem. Phys. 89, 1560 (1988).

[13] T. Helgaker and P. Jørgensen, Theor. Chim. Acta 75, 111 (1989).

[14] H. Koch and P. Jørgensen, J. Chem. Phys. 93, 3333 (1990).

[15] T. B. Pedersen and H. Koch, J. Chem. Phys. 106, 8059 (1997).

16] O. Christiansen, P. Jørgensen, and C. HÄttig, Int. J. Quantum Chem. 68, 1 (1998).

17] O. Christiansen, H. Кoch, and P. Jørgensen, Chem. Phys. Lett. 243, 409 (1995).

[18]R. J. Wheatney, J. Comput. Chem. 29, 445 (2009).

[19]K. Hald, F. Pawłowski, P. Jørgensen, and C. Hättig, J. Chem. Phys. 118, 1292 (2003).

[20]R. F. Hammond, W. A. De Jong, and K. Kowalski, J. Chem. Phys. 128, 224102 (2009).

[21] M. Kallay and J. Gauss, J. Mol. Struct. (THEOCHEM) 768, 71 (2006).

[22] J. Oddershede, Adv. Quant. Chem. 11, 275 (1978).

[23] R. Kobayashi, H. Koch, and P. Jørgensen, Chem. Phys. Lett. 219, 30 (1994).

[24] O. Christiansen, A. Halkier, H. Koch, and P. Jørgensen, J. Chem. Phys. 108, 2801 (1998).

[25]C. Hättig, O. Christiansen, S. Coriani, and P. Jørgensen, J. Chem. Phys. 109, 9237 (1998).

[26]H. Larsen, J. Olsen, C. Ḧ̈ttig, and P. Jørgensen, J. Chem. Phys. 111, 1917 (1999).

27] S. Coriani, A. Halkier, and P. Jørgensen, J. Chem. Phys. 113, 1688 (2000).

[28] M. Jaszunski, A. Rizzo, and P. Jørgensen, Theor. Chem. Acc. 106, 251 (2001).

[29] M. J. Paterson, O. Christiansen, F. Pawłowski, P. Jørgensen, C. Hättig, T. Helgaker, and P. SAŁeK, J. Chem. Phys. 124, 054322 (2006).

[30]K. Kowalski, J. R. Hammond, W. A. De Jong, and A. J. Sadlej, J. Chem. Phys. 129, 226101 (2008).

[31]J. Noga and M. Urban, Theor. Chim. Acta 73, 291 (1988).

[32] B. Jeziorski and R. Moszynski, Int. J. Quantum Chem. 48, 161 (1993).

[33]R. Moszynski, P. S. Żuchowski, and B. Jeziorski, Coll. Czech. Chem. Commun. 70, 1109 (2005).

[34] T. Korona, M. Przybytek, and B. Jeziorski, Mol. Phys. 104, 2303 (2006).

[35] T. Korona and B. Jeziorski, J. Chem. Phys. 125, 184109 (2006).

[36] T. Korona, Phys. Chem. Chem. Phys. 10, 5698 (2008).

[37] T. Korona, J. Chem. Phys. 122, 224104 (2008).

[38] T. Korona, Phys. Chem. Chem. Phys. 10, 6509 (2008).

[39] T. Korona, J. Chem. Theory Comp. 5, 2663 (2009).

[40]B. Jeziorski, R. Moszynski, and K. Szalewicz, Chem. Rev. 94, 1887 (1994).

41] O. Christiansen, H. Koch, and P. Jørgensen, J. Chem. Phys. 103, 7429 (1995).

[42]R. Moszynski, B. Jeziorski, and K. Szalewicz, J. Chem. Phys. 100, 1312 (1994).

[43]H. J. Monkhorst, B. Jeziorski, and F. E. Harris, Phys. Rev. A 23, 1639 (1981).

[44]B. H. Brandow, Adv. Quant. Chem. 10, 187 (1977).

[45] P. E. S. Wormer and H. Hettema, J. Chem. Phys. 97, 5592 (1992).

[46]N. M. Hugenholtz, Physica (Utrecht) 23, 481 (1957). 
Table 1. Components of the TD-CCSDT propagator giving a contribution in a low $W$ order. For the components containing $\bar{Y}$ and $\Omega(\omega)$ and for the term 10 the respective op.ex. terms should be added (the op.ex. term is obtained by a simultaneous exchange of the operators $X$ and $Y$ and replacing $\omega$ by $-\omega)$. The lowest MP order to which a given term contributes is presented in the second column. The last column contains an information if these terms are fully included on the CCSD level (the abbreviation $Y$ denotes that the whole term is included, $N$ - that the whole term is missing, $P$ - that some parts of this term are missing).

\begin{tabular}{rclc}
\hline Nr & MP order & Term & in TD-CCSD? \\
\hline 1 & 0 & $\left\langle Y \Omega_{1}(\omega)\right\rangle$ & $\mathrm{P}$ \\
2 & 1 & $\left\langle\left[\left[W, \Omega_{1}(\omega)\right], \Xi_{1}(-\omega)\right]\right\rangle$ & $\mathrm{Y}$ \\
3 & 2 & $\left\langle\Lambda_{1} \mid\left[\bar{Y}, \Omega_{1}(\omega)\right]\right\rangle$ & $\mathrm{Y}$ \\
4 & 2 & $\left\langle\Lambda_{2} \mid\left[\bar{Y}, \Omega_{2}(\omega)\right]\right\rangle$ & $\mathrm{P}$ \\
5 & 2 & $\left\langle\Lambda_{2} \mid\left[\bar{Y}, \Omega_{1}(\omega)\right]\right\rangle$ & $\mathrm{Y}$ \\
6 & 2 & $\left\langle\Lambda_{2} \mid\left[\left[\bar{W}, \Omega_{1}(\omega)\right], \Xi_{1}(-\omega)\right]\right\rangle$ & $\mathrm{Y}$ \\
7 & 3 & $\left\langle\Lambda_{1} \mid\left[\bar{Y}, \Omega_{2}(\omega)\right]\right\rangle$ & $\mathrm{Y}$ \\
8 & 3 & $\left\langle\Lambda_{2} \mid\left[\bar{Y}, \Omega_{3}(\omega)\right]\right\rangle$ & $\mathrm{N}$ \\
9 & 3 & $\left\langle\Lambda_{1} \mid\left[\left[\bar{W}, \Omega_{1}(\omega)\right], \Xi_{1}(-\omega)\right]\right\rangle$ & $\mathrm{Y}$ \\
10 & 3 & $\left\langle\Lambda_{2} \mid\left[\left[\bar{W}, \Omega_{1}(\omega)\right], \Xi_{2}(-\omega)\right]\right\rangle$ & $\mathrm{Y}$ \\
\hline
\end{tabular}

Table 2. Components of the XCCSDT propagator giving a contribution in a low $W$ order. For each term the corresponding g.c.c. counterpart should be added. For explanations of the second and fourth columns see Table 1.

\begin{tabular}{rclc}
\hline $\mathrm{Nr}$ & MP order & Term & in XCCSD? \\
\hline 1 & 0 & $\left\langle Y \mid \Omega_{1}(\omega)\right\rangle$ & $\mathrm{P}$ \\
2 & 1 & $\left\langle\left[Y, S_{2}\right] \mid \Omega_{1}(\omega)\right\rangle$ & $\mathrm{P}$ \\
3 & 2 & $\left\langle\left[Y, S_{1}\right] \mid \Omega_{1}(\omega)\right\rangle$ & $\mathrm{P}$ \\
4 & 2 & $\left\langle\left[Y, S_{2}\right] \mid \Omega_{2}(\omega)\right\rangle$ & $\mathrm{P}$ \\
5 & 2 & $\left\langle\left[T_{2}^{\dagger}, Y\right], S_{2}\right]\left|\Omega_{1}(\omega)\right\rangle$ & $\mathrm{Y}$ \\
6 & 3 & $\left.\left.\langle Y| S_{1}^{\dagger}, \Omega_{2}(\omega)\right]\right\rangle$ & $\mathrm{Y}$ \\
7 & 3 & $\left\langle Y \mid\left[S_{2}^{\dagger}, \Omega_{3}(\omega)\right]\right\rangle$ & $\mathrm{N}$ \\
8 & 3 & $\left\langle\left[\left[T_{1}^{\dagger}, Y\right], S_{2}\right] \mid \Omega_{1}(\omega)\right\rangle$ & $\mathrm{Y}$ \\
9 & 3 & $\left\langle\left[T_{2}^{\dagger}, Y\right], S_{3}\right]\left|\Omega_{1}(\omega)\right\rangle$ & $\mathrm{N}$ \\
10 & 3 & $\left\langle\left[Y, S_{3}\right] \mid \Omega_{2}(\omega)\right\rangle$ & $\mathrm{N}$ \\
\hline
\end{tabular}


Table 3. Terms arising from triple excitation operators of the XCCSDT polarization propagator contributing at the MP3 order. For each term its g.c.c. counterpart should be added. Terms Ia, Ib, Ic, and Id are obtained by inserting the definition of $\Omega_{1}^{(3 t)}(\omega)$ from Eq. (21) (the order of terms from Eq. (21) is preserved). Similarly, an insertion of $\Omega_{2}^{(1)}(\omega)$ (Eq. (16)) leads to the terms IVa and IVb.

\begin{tabular}{clc}
\hline Contribution from triples & Label & Term from Table 2 \\
\hline$\left\langle Y \mid \Omega_{1}^{(3 t)}(\omega)\right\rangle$ & Ia, Ib, Ic, Id & 1 \\
$\left\langle\left[Y, T_{1}^{(3 t)}\right] \mid \Omega_{1}^{(0)}(\omega)\right\rangle$ & II & 3 \\
$\left\langle\left[Y, \widehat{\mathcal{P}}_{1}\left(\left[T_{2}^{(1) \dagger}, T_{3}^{(2)}\right]\right)\right] \mid \Omega_{1}^{(0)}(\omega)\right\rangle$ & III & 3 \\
$\left\langle\left[Y, T_{3}^{(2)}\right] \mid \Omega_{2}^{(1)}(\omega)\right\rangle$ & IVa, IVb & 10 \\
$\left\langle\left[Y, T_{2}^{(3 t)}\right] \mid \Omega_{1}^{(0)}(\omega)\right\rangle$ & V & 2 \\
$\left\langle\left[Y, T_{2}^{(1)}\right] \mid \Omega_{2}^{(2 t)}(\omega)\right\rangle$ & VI & 2 \\
$\left\langle T_{2}^{(1)} \mid\left[Y, \Omega_{3}^{(2)}(\omega)\right]\right\rangle$ & VII & 7 \\
$\left\langle\left[\left[T_{2}^{(1) \dagger}, Y\right], T_{3}^{(2)}\right] \mid \Omega_{1}^{(0)}(\omega)\right\rangle$ & VIII & 9 \\
\hline
\end{tabular}

Table 4. Terms arising from triple excitation operators of the TD-CCSDT polarization propagator contributing at the MP3 order. For each term its op.ex. counterpart should be added.

\begin{tabular}{clc}
\hline Contribution from triples & Label & Term from Table 1 \\
\hline$\left\langle Y \mid \Omega_{1}^{(3 t)}(\omega)\right\rangle$ & Ia, Ib, Ic, Id & 1 \\
$\left\langle T_{2}^{(1)} \mid\left[Y, \Omega_{2}^{(2 t)}(\omega)\right]\right\rangle$ & VI & 4 \\
$\left\langle T_{2}^{(1)} \mid\left[Y, \Omega_{3}^{(2)}(\omega)\right]\right\rangle$ & VII & 8 \\
\hline
\end{tabular}




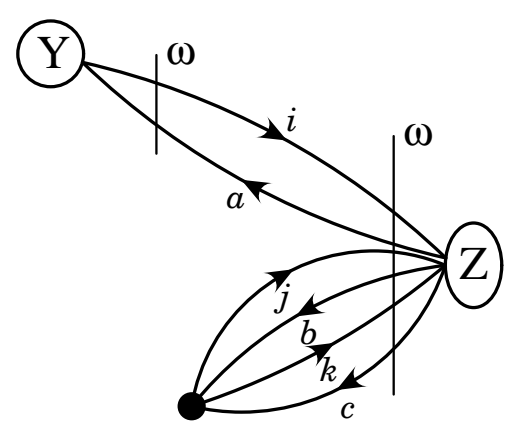

(a)

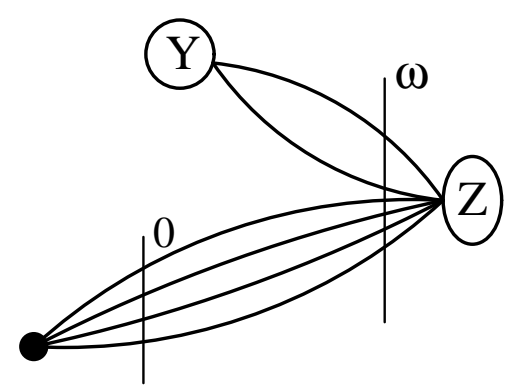

(b)

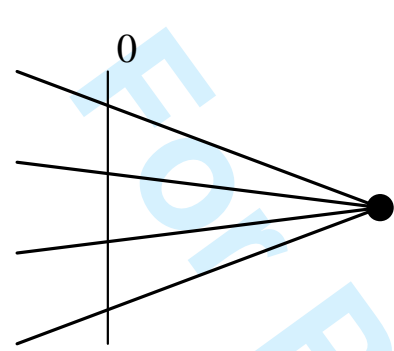

(c)

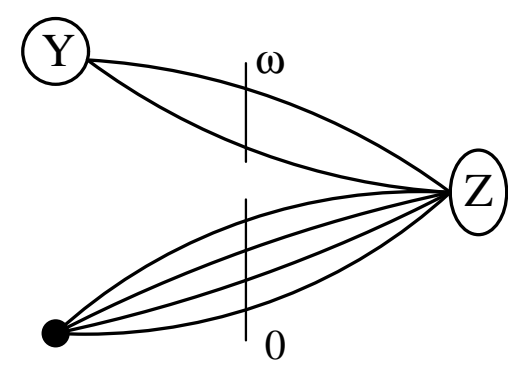

(d)

Figure 1. Diagrams replacing the derivation steps from Eq. (24) to Eq. (29): a) the term Id , i.e. $\left\langle Y \mid \widehat{\mathcal{R}}_{1, \omega}\left(\left[W, \Omega_{3}^{(2)}(\omega)\right]\right)\right\rangle$, where Eq. (22) has been utilized for $\Omega_{3}^{(2)}(\omega)$; b) its guessed counterpart $\left\langle T_{2}^{(1)} \mid\left[Y, \Omega_{3}^{(2)}(\omega)\right]\right\rangle$, which can be identified as the term VII; c) the $T_{2}^{(1)}$ operator, present in the diagram drawn on Figure $1(\mathrm{~b})$; d) the final result after the elimination of the triple energy denominator, i.e. $\left\langle\widehat{\mathcal{R}}_{1, \omega^{*}}(Y) \widehat{\mathcal{R}}_{2,0}(W) \mid Z\right\rangle$;

Note that the old Figures 1 and 2 have been removed 


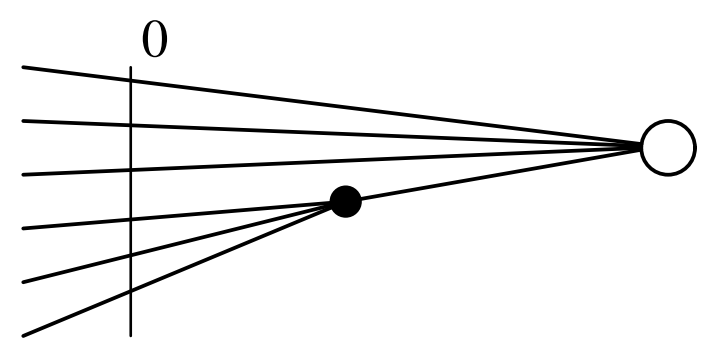

(a)

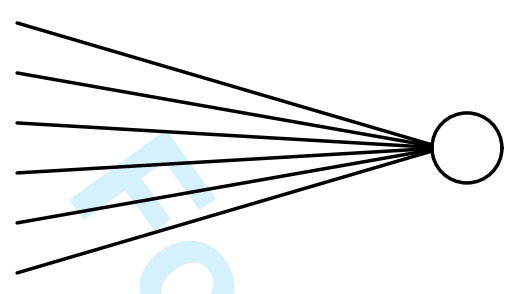

(b)

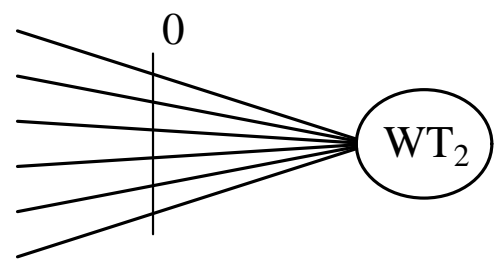

(c)

Figure 2. Diagrammatic representations of the $T_{3}^{(2)}$ operator.

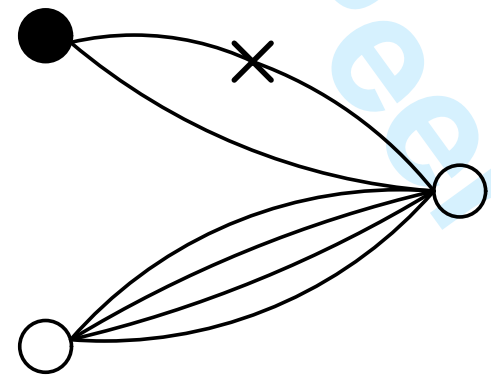

(a)

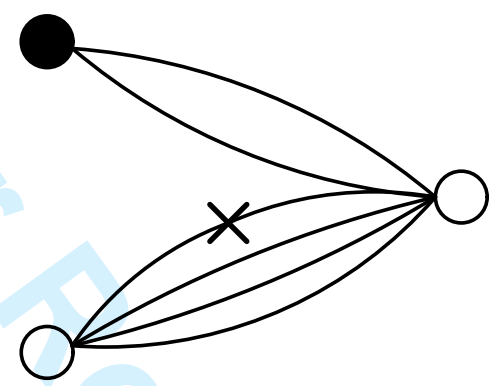

(b)

Figure 3. The r.h.s. of Eq. (31): a) $\left\langle\widehat{\mathcal{P}}_{1}\left(X \Xi_{1}^{(0)}\left(\omega^{*}\right)\right) T_{2}^{(1)} \mid T_{3}^{(2)}\right\rangle$; b) $\left\langle\Xi_{1}^{(0)}\left(\omega^{*}\right) \widehat{\mathcal{P}}_{2}\left(\left[X, T_{2}^{(1)}\right]\right) \mid T_{3}^{(2)}\right\rangle$. 


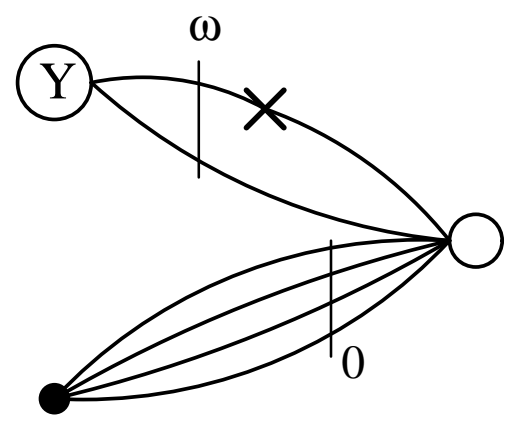

(a)

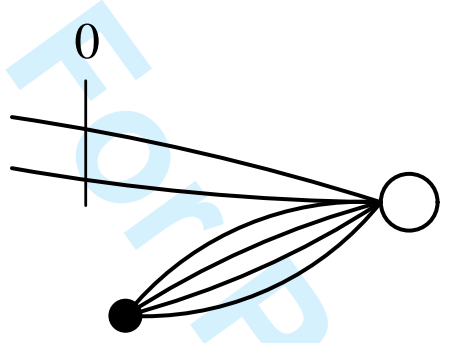

(c)

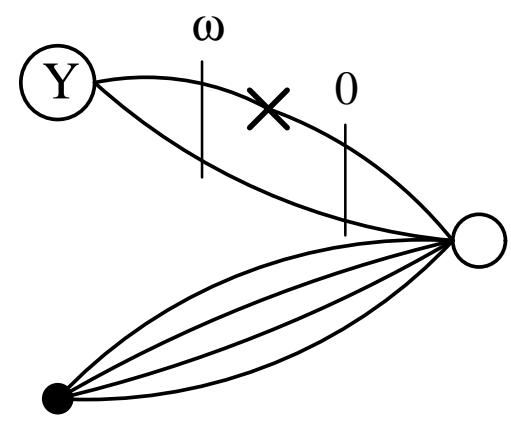

(b)

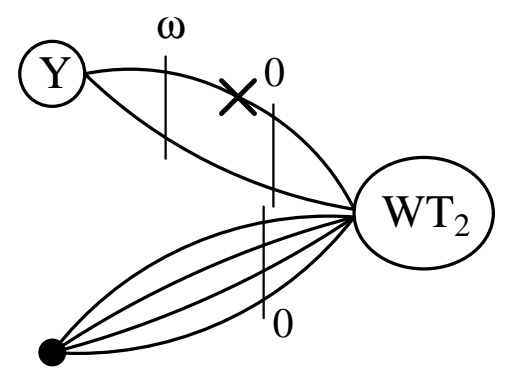

(d)

Figure 4. Diagrams replacing the derivation steps from Eq. (32) to Eq. (33): a) the diagram from Figure 3(a) with the expanded forms of $\Xi_{1}^{(0)}\left(\omega^{*}\right)$ and $T_{2}^{(1)}$ (see Eq. (16)); b) the complementary diagram, $\left\langle Y \mid \widehat{\mathcal{R}}_{1, \omega}\left(\left[X, T_{1}^{(3 t)}\right]\right)\right\rangle$, (i.e. the term Ia); c) the diagram for the $T_{1}^{(3 t)}$ operator; d) the result of adding of the diagrams $4(\mathrm{a})$ and $4(\mathrm{~b})$, $\left\langle\widehat{\mathcal{R}}_{1,0}\left(X \widehat{\mathcal{R}}_{1, \omega^{*}}(Y)\right) \widehat{\mathcal{R}}_{2,0}(W) \mid\left[W, T_{2}^{(1)}\right]\right\rangle$.

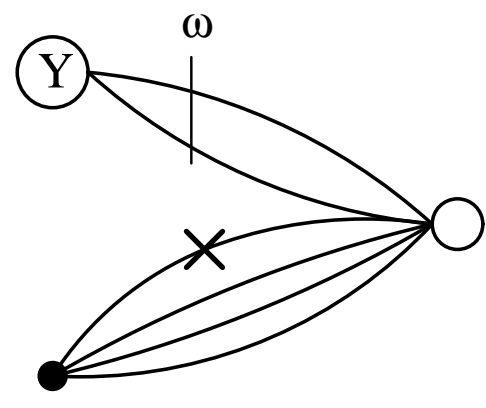

(a)

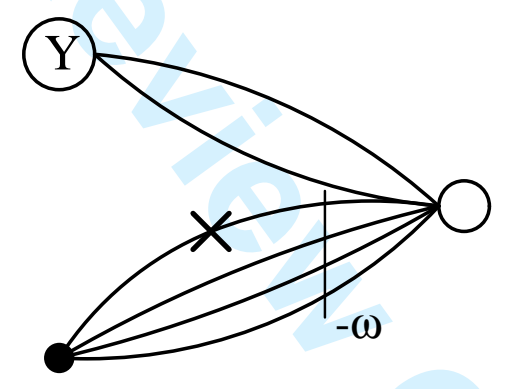

(b)

Figure 5. The diagrams explaining Eqs. (31) and (36): a) the second part of the r.h.s. of Eq. (31) with the expanded form of $\Xi_{1}^{(0)}\left(\omega^{*}\right)$ (see Eq. (16)); b) its counterpart $\left\langle\widehat{\mathcal{R}}_{2,-\omega^{*}}\left(\left[X, T_{2}^{(1)}\right]\right) \mid\left[Y, T_{3}^{(2)}\right]\right\rangle$, which can be identified as g.c.c. of the term IVa. 
1

2

3

4

5

6

7

8

9

10

12

13

14

15

16

17

18

19

20

21

22

23

24

25

26

27

28

29

30

31

32

33

34

35

36

37

38

39

40

41

42

43

44

45

46

47

48

49

50

51

52

53

54

55

56

57

58

59

60

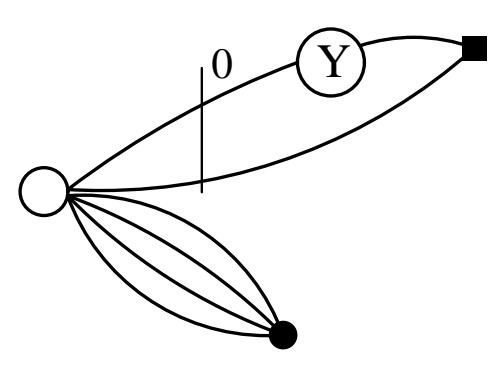

(a)

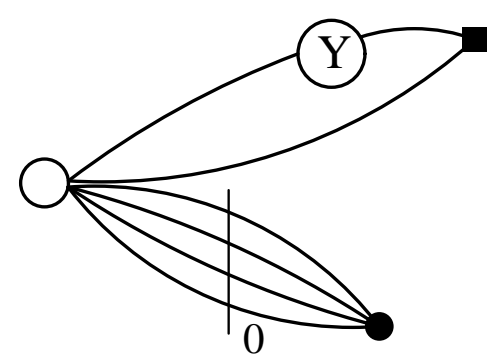

(b)

Figure 6. The diagrams replacing Eqs. (37) to (39): a) the term II, i.e. $\left\langle T_{1}^{(3 t)} \mid Y \Omega_{1}^{(0)}(\omega)\right\rangle$, where the diagrammatic representation of the operator $\left(T_{1}^{(3 t)}\right)^{\dagger}$, $c f$. Figure 4(c), has been utilized; b) the complementary diagram, corresponding to the term III, i.e. $\left\langle T_{3}^{(2)} \mid \widehat{\mathcal{R}}_{2,0}(W) \widehat{\mathcal{P}}_{1}\left(Y \Omega_{1}^{(0)}(\omega)\right)\right\rangle$.

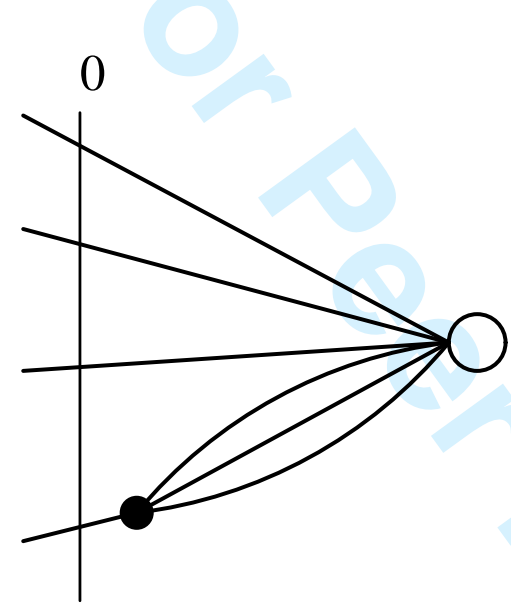

(a) $\omega$

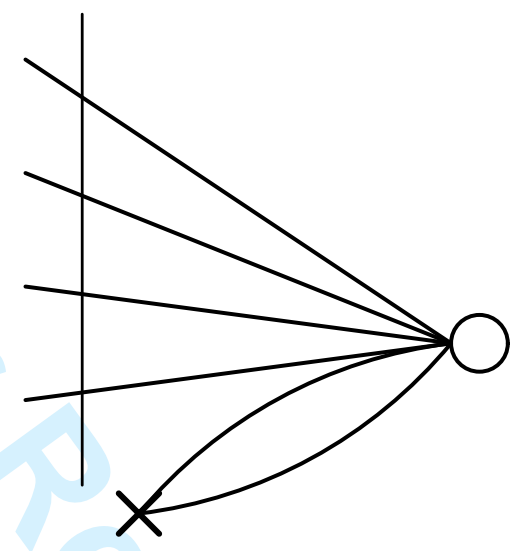

(b)

Figure 7. The operators $T_{2}^{(3 t)}$ (a) and $\Omega_{2}^{(2 t)}(\omega)$ (b), see Eq. (21). 


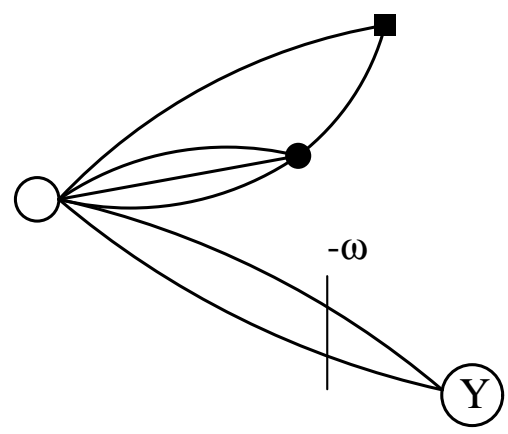

(a)

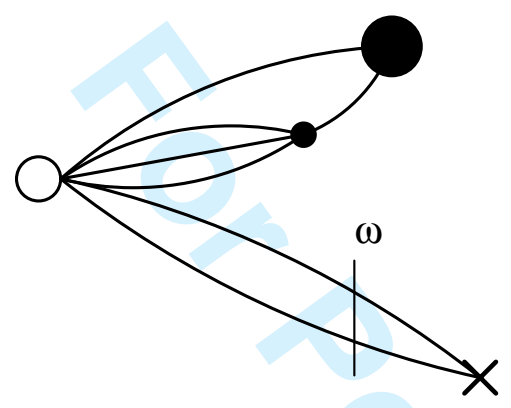

(c)

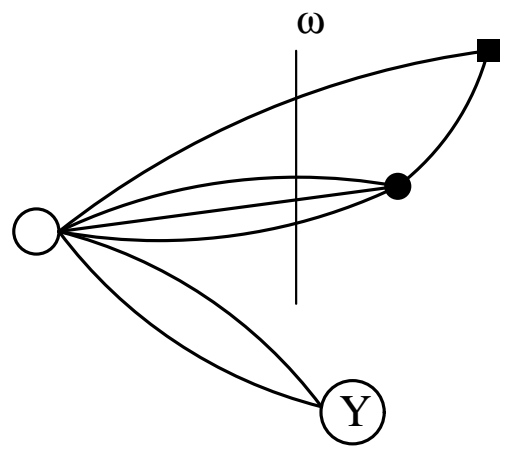

(b)

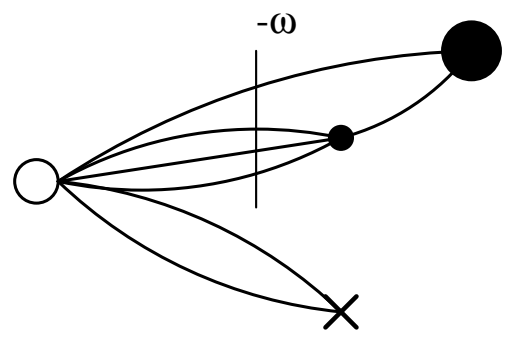

(d)

Figure 8. Diagrams explaining the derivation steps from Eqs. (43)-(47). The first and second terms of the r.h.s. of Eq. (44) are represented by the diagrams: a) the term $\left\langle T_{3}^{(2)} \mid \widehat{\mathcal{P}}_{2}\left(W \Omega_{1}^{(0)}(\omega)\right) \widehat{\mathcal{R}}_{1,-\omega}(Y)\right\rangle$; and c) the term $\left\langle T_{3}^{(2)} \mid \widehat{\mathcal{R}}_{1, \omega}(X) \widehat{\mathcal{P}}_{2}\left(W \Xi_{1}^{(0)}(-\omega)\right)\right\rangle$. The complementary diagrams, shown on the right side, are: b) the term $\left\langle\left[Y, T_{3}^{(2)}\right] \mid \widehat{\mathcal{R}}_{2, \omega}\left(\left[W, \Omega_{1}^{(0)}(\omega)\right]\right)\right\rangle$, identified as the term IVb; d) the term $\left\langle\widehat{\mathcal{R}}_{1,-\omega^{*}}\left(\left[W, \Omega_{2}^{(2 t)}\left(-\omega^{*}\right)\right]\right) \mid Y\right\rangle$, identified as g.c.c. of the term Ic.

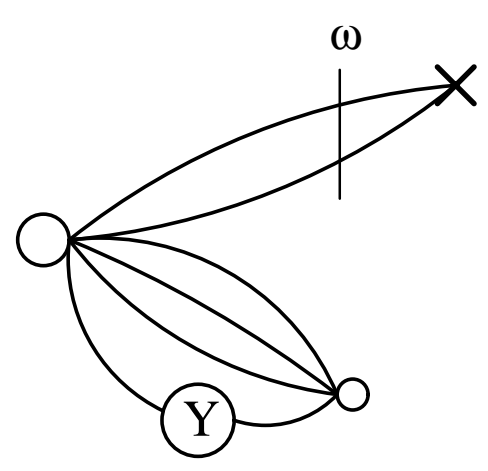

(a)

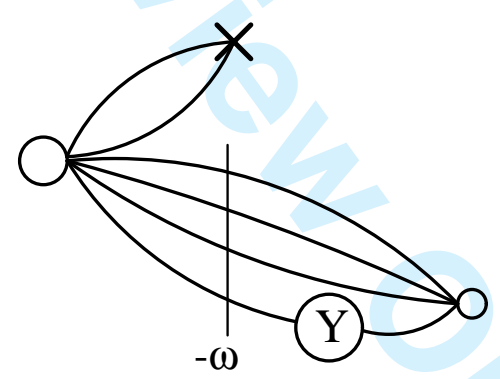

(b)

Figure 9. The diagrams explaining Eqs. (48)-(50): a) the term VIII, $\left\langle T_{3}^{(2)} \mid \Omega_{1}^{(0)}(\omega) \widehat{\mathcal{P}}_{2}\left(\left[Y, T_{2}^{(1)}\right]\right)\right\rangle ;$ b) the complementary diagram, corresponding to the term $\left\langle\Omega_{2}^{(2 t)}\left(-\omega^{*}\right) \mid\left[Y, T_{2}^{(1)}\right]\right\rangle$, i.e. g.c.c. of the term VI. 